\title{
A nationwide survey concerning the mortality and risk of progressing severity due to arterial and venous thromboembolism in inflammatory bowel disease in Japan
}

\author{
Katsuyoshi Ando ${ }^{1} \cdot$ Mikihiro Fujiya $^{1}$ (D) Kenji Watanabe ${ }^{2} \cdot$ Sakiko Hiraoka $^{3}$. \\ Hisashi Shiga $^{4}$ - Shinji Tanaka ${ }^{5}$ Hideki Iijima $^{6}$ - Tsunekazu Mizushima ${ }^{7}$. \\ Taku Kobayashi ${ }^{8}$ - Masakazu Nagahori ${ }^{9}$ Hiroki Ikeuchi' ${ }^{10}$ - Shingo Kato ${ }^{11}$. \\ Takehiro Torisu $^{12}$ - Kiyonori Kobayashi ${ }^{13}$ - Masaaki Higashiyama ${ }^{14}$. \\ Toshiro Fukui ${ }^{15}$. Takashi Kagaya ${ }^{16}$. Motohiro Esaki ${ }^{17} \cdot$ Shunichi Yanai ${ }^{18}$. \\ Daiki Abukawa $^{19} \cdot$ Makoto Naganuma $^{15,20} \cdot$ Satoshi Motoya ${ }^{21}$ - Masayuki Saruta ${ }^{22}$. \\ Shigeki Bamba ${ }^{23}$ - Makoto Sasaki ${ }^{24}$ Kazuhiko Uchiyama ${ }^{25}$ - Katsuyuki Fukuda ${ }^{26}$. \\ Hideo Suzuki ${ }^{27}$ - Hiroshi Nakase ${ }^{28}$ - Toshiaki Shimizu ${ }^{29}$ - Masahiro Iizuka ${ }^{30}$. \\ Mamoru Watanabe $^{31} \cdot$ Yasuo Suzuki $^{32}$ - Tadakazu Hisamatsu ${ }^{33}$
}

Received: 8 July 2021/Accepted: 15 September 2021/Published online: 5 October 2021

(C) The Author(s) 2021

\begin{abstract}
Background The mortality and risk factors of severe disease and death due to arterial and venous thromboembolism (ATE and VTE, respectively) in patients with inflammatory bowel disease (IBD) remain unclear, especially in Asia.
\end{abstract}

Mikihiro Fujiya

fjym@asahikawa-med.ac.jp

1 Gastroenterology and Endoscopy, Division of Metabolism and Biosystemic Science, Gastroenterology, and Hematology/Oncology, Department of Medicine, Asahikawa Medical University, 2-1 Midorigaoka-higashi, Asahikawa, Hokkaido 078-8510, Japan

2 Center for Inflammatory Bowel Disease, Division of Internal Medicine, Hyogo College of Medicine, Hyogo, Japan

3 Department of Gastroenterology and Hepatology, Graduate School of Medicine, Dentistry and Pharmaceutical Sciences, Okayama University, Okayama, Japan

4 Division of Gastroenterology, Tohoku University Graduate School of Medicine, Miyagi, Japan

5 Department of Endoscopy and Medicine, Hiroshima University Hospital, Hiroshima, Japan

6 Department of Gastroenterology and Hepatology, Osaka University Graduate School of Medicine, Osaka, Japan

7 Department of Gastroenterological Surgery and Department of Therapeutics for Inflammatory Bowel Diseases, Graduate School of Medicine, Osaka University, Osaka, Japan
Aims This study aimed to reveal the mortality and risk factors of TE in IBD patients in Japan.

Methods In the primary surveillance, responses to questionnaires regarding the number of cases of severe TE and TE-associated death in IBD patients in a span of over the past 10 years were obtained from 32 institutions in Japan. In the secondary surveillance, detailed data about IBD

8 Center for Advanced IBD Research and Treatment, Kitasato University Kitasato Institute Hospital, Tokyo, Japan

9 Department of Gastroenterology and Hepatology, Tokyo Medical and Dental University, Tokyo, Japan

10 Department of Inflammatory Bowel Disease, Division of Surgery, Hyogo College of Medicine, Hyogo, Japan

11 Department of Gastroenterology and Hepatology, Saitama Medical Centre, Saitama Medical University, Saitama, Japan

12 Department of Medicine and Clinical Science, Graduate School of Medical Sciences, Kyushu University, Fukuoka, Japan

13 Research and Development Center for New Medical Frontiers, Kitasato University School of Medicine, Kanagawa, Japan

14 Division of Gastroenterology and Hepatology, Department of Internal Medicine, National Defense Medical College, Saitama, Japan

15 The Third Department of Internal Medicine, Division of Gastroenterology and Hepatology, Kansai Medical University, Osaka, Japan

16 Departments of Gastroenterology, National Hospital Organization Kanazawa Medical Center, Kanazawa, Japan 
patients with TE were collected. The characteristics, laboratory data, therapy status, and situation at the time of TE development were retrospectively collected, and the data were compared between the patients with and without severe TE and TE-associated death.

Results The incidence of TE was $1.89 \%$ among 31,940 IBD patients. The frequencies of severe TE and TE-associated mortality were $10.7 \%$ and $1.0 \%$ among the total IBD and TE with IBD patients, respectively. The only risk factor for severe ATE and ATE-associated death was ischemic heart disease. The independent risk factors for severe VTE and VTE-associated death were age ( $\leq 45$ years old), the site of VTE, and disease severity, with anti-TNF therapy as a potential negative risk factor. Patients with severe VTE had a high risk of developing persistent VTE and sequelae.

Conclusion Unlike ATE, the incidence of VTE was comparable in Asian and Western countries. Therapeutic and prophylactic strategies for managing IBD-associated TE in Asia are urgently needed.

Keywords Venous thromboembolism - Arterial thromboembolism - Inflammatory bowel disease . Mortality $\cdot$ Severity

\section{Introduction}

Inflammatory bowel disease (IBD) is a chronic and relapsing intestinal and systemic inflammatory disorder that has two main phonotypes: ulcerative colitis (UC) and Crohn's disease (CD). Extraintestinal manifestations, as

17 Division of Gastroenterology, Department of Internal Medicine, Faculty of Medicine, Saga University, Saga, Japan

18 Division of Gastroenterology, Department of Internal Medicine, School of Medicine, Iwate Medical University, Iwate, Japan

19 Division of General Pediatrics and Gastroenterology, Miyagi Children's Hospital, Miyagi, Japan

20 Division of Gastroenterology and Hepatology, School of Medicine, Keio University, Tokyo, Japan

21 IBD Center, Hokkaido Prefectural Welfare Federation of Agricultural Cooperative, Sapporo-Kosei General Hospital, Hokkaido, Japan

22 Division of Gastroenterology and Hepatology, Department of Internal Medicine, The Jikei University School of Medicine, Tokyo, Japan

23 Division of Digestive Endoscopy, Shiga University of Medical Science, Shiga, Japan

24 Division of Gastroenterology, Department of Internal Medicine, Aichi Medical University School of Medicine, Aichi, Japan well as intestinal complications such as stenosis and abscess formation, frequently occur in IBD. Thromboembolism (TE) is a serious extraintestinal manifestation [1-4] that has been suggested to correlate with chronic inflammation, hypercoagulability, endovascular dysfunction, and atherosclerosis [5, 6]. Indeed, large-scale cohort studies from Western countries have shown that the incidence of venous thromboembolism (VTE) in IBD patients was twoto threefold greater than that in non-IBD controls [7, 8].

In recent years, several reports have suggested that IBD patients have an increased risk of arterial thromboembolism (ATE), including ischemic heart disease (IHD) and cerebrovascular disease (CVD), compared with non-IBD patients [9-11]. Notably, ATE and VTE are regarded as life-threatening complications of IBD in clinical practice due to the potential need for invasive therapy (admission to the intensive care unit, catheter intervention, and surgery) and consequences of severe organ failure and death [1]. In previous studies, the mortality rate of IBD-associated VTE has been reported to range from $10.7 \%$ to $25 \%$ [12-14]; however, most of these reports included data from before the start of biologics treatment (1970s to 2000s), regions other than Asia and races other than Asians. Nguyen et al. reported that the in-hospital mortality rate of IBD patients with VTE was 2.5- and 2.1-times greater than that in IBD patients without VTE and non-IBD patients with VTE, respectively [15]. Furthermore, in that study, the risk factors of in-hospital death in patients with IBD were suggested to be UC, an older age, presence of comorbidities, health insurance (Medicare or Medicaid), and IBD-related surgery in addition to VTE [15]. However, these risk

25 Molecular Gastroenterology and Hepatology, Graduate School of Medical Science, Kyoto Prefectural University of Medicine, Kyoto, Japan

26 Department of Gastroenterology, St Luke's International Hospital, Tokyo, Japan

27 Department of Gastroenterology, Graduate School of Institute Clinical Medicine, University of Tsukuba, Ibaraki, Japan

28 Department of Gastroenterology and Hepatology, Sapporo Medical University School of Medicine, Hokkaido, Japan

29 Department of Pediatrics and Adolescent Medicine, Juntendo University Graduate School of Medicine, Tokyo, Japan

30 Health Care Center, Akita Red Cross Hospital, Akita, Japan

31 Institute of Innovation Advancement, Tokyo Medical and Dental University, Tokyo, Japan

32 Department of Internal Medicine, Toho University Medical Center Sakura Hospital, Chiba, Japan

33 Department of Gastroenterology and Hepatology, Kyorin University School of Medicine, Tokyo, Japan 
factors for in-hospital mortality in IBD were analyzed in hospitalized patients who died of IBD, so these were not risk factors for death in IBD patients with VTE. Conversely, according to two meta-analyses, the mortality of IBD patients with ATE was comparable to that of non-IBD controls with ATE $[8,16]$. It has also been reported that the mortality of ATE was increased in specific subgroups of IBD; those with colonic-type CD have a risk of cardiovascular disease, women $\geq 40$ years old have a risk of myocardial infarction, and women $<40$ years old have a risk of stroke and related diseases [17-19]. These data from Western countries clearly show the high incidences of TE and TE-associated disease severity in IBD patients.

In Asian countries, a multi-national study revealed that the incidence of VTE in hospitalized IBD patients was 2.27 times greater than that in non-IBD controls, [20] although the sample size of that study was much smaller than those in studies from Western countries [4, 7]. No large-scale nationwide study concerning the incidence and risk factors for TE-associated severity and death in IBD patients has been reported from Eastern countries, and the incidences and mortalities of ATE and VTE in the general population differ between Eastern and Western countries [21, 22]. Pharmacological prophylaxis for VTE has been recommended for all hospitalized IBD patients in Western countries according to various guidelines and consensus statements [7, 23, 24]; however, in Asia, pharmacological prophylaxis has not been described due to a lack of evidence. Therefore, clarifying the incidence of TE and risk factors of TE-associated severity and death in IBD patients in Eastern countries is necessary to establish an appropriate risk classification of VTE and reasonable strategies for screening and prophylaxis.

The present nationwide surveillance aimed to determine the incidence of $\mathrm{TE}$ and risk factors of TE-associated severity and death in an Asian population.

\section{Methods}

\section{Primary surveillance for the incidence of TE and TE-associated severity and death in IBD patients}

A questionnaire concerning the incidence of TE, ATE, VTE, and severe TE and TE-associated death in IBD patients was distributed to participating institutions on the Research Committee of Inflammatory Bowel Disease the Ministry of Health and Welfare of Japan, and 32 institutions responded to the questionnaire. The subjects of the survey were IBD patients who visited the participating institutions from January 2008 to December 2017.
The questionnaire inquired about the following: (1) the total number of IBD patients (UC and CD, respectively) in each institute, (2) the number of IBD patients (UC and CD, respectively) who developed ATE and VTE among the patients in each institute, and (3) the number of IBD patients who developed severe TE and/or TE-associated death among the patients in item 2 . The number of severe and non-severe ATE/VTE patients that did and did not result in death was surveyed with a database and chart review in each institution. ATE and VTE were confirmed by imaging, regardless of the presence of symptoms. ATE consisted of the following: CVD, such as cerebral thrombosis and infarction; IHD, such as angina pectoris and acute myocardial infarction; limbs and peripheral ATE; and mesenteric ATE. VTE consisted of the following: deep venous thromboembolism (DVT) in the lower limbs (L), pulmonary arterial thromboembolism (PE) with or without DVT in L, cerebral venous sinus thrombosis (CVS), portal and mesenteric VTE (PMV), and catheter-related thrombosis (CR). Severe TE was defined as cases requiring invasive therapy, including catheter intervention, surgery, and intensive care, and leading to severe organ failure, such as heart, respiratory, liver, and renal failure due to TE.

\section{Secondary surveillance for the risk factors of severe TE and TE-associated death in IBD patients}

The secondary surveillance was performed as a retrospective study to determine the features and risk factors of IBD patients who developed severe TE and TE-associated death. Following the primary surveillance, the details of all IBD cases with ATE and VTE, including those that developed severe TE and death, during the period covered by the primary surveillance were collected by a database and chart review in each institute. The definitions of ATE, VTE, and severe TE were similar to those used in the primary surveillance. The characteristics, activity of IBD, laboratory data and medical therapy for IBD at the time of the development of VTE, site of thrombosis, treatment for ATE and VTE, and the outcomes, including the severity, sequelae, and death due to ATE and VTE, were retrospectively collected in IBD patients who developed ATE or VTE. The data in cases with severe TE and TE-associated death were compared with those in cases without severe TE and TE-associated death.

\section{Endpoints}

The primary endpoint of this study was the incidence of severe TE and TE-associated mortality in IBD patients. The secondary endpoints were as follows: the incidence of severe TE and TE-associated mortality at each site of TE development, the risk factors for TE-associated severity 
and death in IBD patients, the period from the onset of TE until severe disease and death, therapy and outcomes after developing TE, and hemorrhagic complications of antithrombotic and fibrinolytic therapy.

The study protocol was approved by the ethics committees of Asahikawa Medical University (authorization number 18139) and other participating institutions and was registered with the University Hospital Medical Information Network (UMIN) Center (UMIN registry number 000030524). Informed consent was obtained by announcing this study on the Internet and providing the opportunity to opt out.

\section{Data collection}

The following patient characteristics were collected: age, sex, height, body weight, body mass index (BMI), disease duration, type of IBD (UC or CD), type of disease extension (UC: Montreal classification; E1, 2, 3, CD: Montreal classification; L1, 2, 3, 4), disease activity (UC: partial Mayo score [pMayo], CD: Crohn's disease activity index [CDAI]; clinical remission was defined as pMayo $\leq 2$ or $\mathrm{CDAI} \leq 150$, mild activity was defined as pMayo $3-5$ or CDAI 151-219, and moderate to severe activity was defined as pMayo $\geq 6$ or $\mathrm{CDAI} \geq 220$ ), extraintestinal manifestations, history of smoking and alcohol consumption, history of thrombosis and thrombotic events, intake of antithrombotic drugs, presence or absence of central venous catheter [CVC], medications (5-aminosalicylate [5ASA], prednisolone [PSL], immunomodulators [IM], calcineurin inhibitor [CNI], anti-TNF- $\alpha$ antibody, ustekinumab [UST], vedolizumab [VDZ], Janus kinase [JAK] inhibitor, and cytapheresis), history of bowel surgery, systemic comorbidities (diabetes mellitus [DM], hypertension [HT], dyslipidemia [DLp], heart and pulmonary disease, renal dysfunction, neuromuscular disease, and malignancy), and presence or absence of a long-term bedridden status (defined as performance status $\geq 2$ ).

Data on the following conditions at the time of ATE and VTE development and therapy toward ATE and VTE were also collected: ambulatory or hospitalized at the time of ATE or VTE development, hospitalization period until the development of ATE or VTE, site of ATE (CVD, IHD, limbs and peripheral ATE, mesenteric ATE, and others), site of VTE (DVT in L, pulmonary ATE, CVS, PVM, catheter-related thrombosis, and others), presence or absence of TE-associated severity or death, period until development of severe disease or death, therapy for ATE and VTE (antithrombotic therapy, fibrinolytic therapy, inferior vena cava filter, catheter intervention, surgery, physical therapy, or observation only), presence or absence of hemorrhagic complications of antithrombotic and fibrinolytic therapy, and outcomes after therapy (improvement with elimination or retention of thrombosis, retention of sequelae, death, or uncertain).

The following laboratory data were also recorded at admission or before the onset of TE (data just before diagnosing TE): white blood cell and platelet counts, serum levels of hemoglobin, C-reactive protein, total protein, albumin, blood urea nitrogen, total cholesterol, triglyceride, and hemoglobin A1c, erythrocyte sedimentation rate (per hour), fibrinogen level, antithrombin III (AT-III), international normalized ratio of prothrombin time (PTINR), active partial thromboplastin time (APTT), D-dimer, fibrin degradation product (FDP), and thrombin-antithrombin complex (TAT).

\section{Statistical analyses}

All statistical analyses were performed using the SPSS software program. Fisher's exact test was used to compare the incidence of ATE and VTE between two or more groups. The incidence and proportion among three or more groups were compared using Fisher's exact test with Holm's correction. An unpaired $t$-test and Fisher's exact test were used to compare patient characteristics and laboratory data between IBD patients with and without severe disease and death. The cutoff values of the items with significant differences were determined by a receiver operating characteristics analysis. A logistic regression analysis was used to identify the independent risk factors leading to severe disease and death in IBD with ATE and VTE, which were detected by entering the significant factors revealed in the univariate analysis into a multivariate model. A $p$ value $<0.05$ was considered statistically significant.

\section{Results}

\section{Primary surveillance}

A total of 31,940 IBD patients $(21,186$ and 10,754 with UC and $\mathrm{CD}$, respectively) who visited the 32 participating institutions within 10 years (2008-2017) were enrolled in this study. The number of IBD patients who developed TE was $604(1.89 \%)$, which consisted of $374(1.77 \%)$ and 195 (1.81\%) patients with UC and CD, respectively. The annual incidence rate of TE was 188.8 per 100,000 person-years in IBD, 176.5 per person-year in UC, and 181.3 per personyear in CD. The number of ATE patients with IBD was 278 $(0.87 \%)$, which consisted of $175(0.83 \%)$ and $103(0.96 \%)$ patients with UC and $\mathrm{CD}$, respectively. In addition, the number of VTE patients with IBD was 328 (1.03\%), which consisted of $202(0.95 \%)$ and $91(0.84 \%)$ patients with UC and CD, respectively. The incidence of ATE and VTE in 
IBD patients was almost equal, as was that between UC and $\mathrm{CD}$. The number of patients who developed severe TE and/or TE-associated death was $65(0.20 \%$ of all IBD patients and $10.7 \%$ of IBD patients with ATE and VTE). The number of patients with TE-associated death was six $(0.019 \%$ of all IBD patients and $1.0 \%$ of IBD patients with TE). All six patients had UC $(0.028 \%$ of all UC patients and $1.6 \%$ of UC patients who developed TE; among these, two had ATE $(0.0094 \%$ of all UC patients and $1.14 \%$ of UC patients with ATE) and four had VTE $(0.019 \%$ of all UC patients and $1.98 \%$ of UC patients with VTE). Among all IBD patients, the estimated annual rate of severe TE and/or TE-associated death was 20.3 per 100,000 IBD patient-years; the estimated annual mortality due to TE was calculated as 1.88 (0.63 with ATE and 1.25 with VTE), whereas it was 2.83 among UC patients (0.94 with ATE and 1.89 with VTE) per 100,000 patient-years (Table 1).

\section{Secondary surveillance}

The detailed data of 215 IBD patients with ATE $(\mathrm{n}=48)$ and VTE $(n=167)$ during the period of the primary surveillance were collected and analyzed in the secondary surveillance.

\section{ATE}

A total of 48 IBD patients who developed ATE were examined. The demographics of the patients with IBD are shown in Table 2. The average age at onset of ATE was 56.3 years old, and $83.3 \%(n=40)$ of the patients with ATE were classified as A3 under the Montreal classification. The proportion of males $(n=33,68.8 \%)$ was about twice as high as that of females $(n=15,31.2 \%)$. The proportion of UC cases $(n=41,85.4 \%)$ was higher than that of $\mathrm{CD}(n=7,14.6 \%)$, and pancolitis-type (E3) UC accounted for $73.1 \%$ of all UC cases. The average disease duration until the development of ATE was 124.2 months. The proportion of patients who were current $(n=17)$ or previous $(n=5)$ smokers was $45.8 \%$. The proportion of patients with comorbidities was $62.5 \%(\mathrm{n}=30)$. Most of the patients had cardiovascular risk factors/metabolic diseases (DM: 22.9\%, HT: 27.1\%, DLp: $16.7 \%$, current or a history of cardio- or CVD: $25.0 \%$ ).

The proportion of patients with ATE who were ambulatory $(n=37,77.1 \%)$ was higher than that of patients who were hospitalized $(n=11,27.9 \%)$. The average hospitalization period in patients who developed ATE was 21 days. The average pMayo score in patients with UC was 2.7 , and the proportion of patients with moderate to severe clinical activity was $8.3 \%(n=4)$. The treatment used for IBD was 5 -ASA in 34 patients $(70.8 \%)$, corticosteroids in 12 (25.0\%), IM in $13(27.1 \%)$, CNI in 1 (2.1\%), anti-TNF antibody in $5(10.4 \%)$, JAK inhibitor in $2(4.2 \%)$, VDZ in 1 (2.1\%), cytapheresis in $1(2.1 \%), \mathrm{CVC}$ in $3(6.3 \%)$, and bowel resection in $4(8.3 \%)$.

The laboratory data before the onset of ATE are shown in Table 2. The number of ATE instances at each site is shown in Fig. 1a. CVD $(n=19,39.6 \%)$ and $\operatorname{IHD}(n=23$, $43.8 \%$ ) accounted for the majority of ATE instances, in contrast to two cases $(4.2 \%)$ in the limbs and peripheral ATE, one case of mesenteric ATE, and three cases at other sites. The total number of patients who developed severe ATE and ATE-associated death was $20(41.7 \%)$ and 2 (4.2\%), respectively, in the cohort collected at the secondary surveillance (Fig. 1b). The proportion of severe ATE and ATE-associated death in IHD (73.9\%, with death accounting for $8.7 \%[n=2]$ ) was significantly higher than that in CVD (26.3\%, with death accounting for $0 \%)$ $(p=0.040)$. The proportion of severe ATE and ATE-associated death in IHD patients $(73.9 \%)$ was also much higher than that in non-IHD patients $(20 \%)(p<0.0001)$ (Fig. 1c). Regarding the age, the proportion of severe ATE and ATE-associated death in patients classified as A1, A2, and A3 was $100 \%$ (1/1), 20\% (1/5), and 50.5\% (20/42, mortality $4.8 \%$ ), respectively (Fig. 1d). Furthermore, when analyzing age groups by decade, the proportion of severe ATE and ATE-associated death in patients in their $\leq 10 \mathrm{~s}$, $20 \mathrm{~s}, 30 \mathrm{~s}, 40 \mathrm{~s}, 50 \mathrm{~s}, 60 \mathrm{~s}, 70 \mathrm{~s}$, and $\geq 80 \mathrm{~s}$ was $100 \%$ (1/ 1), $0 \%$ (0/1), 33.3\% (1/3), 33.3\% (2/6), 52.9\% (8/17), $61.5 \%(8 / 13), 50 \%(2 / 4)$, and $0 \%(0 / 3)$, respectively (Fig. 1e). The proportion of severe ATE and ATE-associated death ranged from 30 to $60 \%$ in patients in their $30 \mathrm{~s}$ to $70 \mathrm{~s}$, with the incidence of ATE peaking in patients in their $50 \mathrm{~s}$ and $60 \mathrm{~s}$; both patients who died due to ATE were in their $60 \mathrm{~s}$ (Fig. 1f). Regarding the development site and age, CVD peaked in patients in their $50 \mathrm{~s}$ and sporadically occurred in any age. In addition, IHD peaked in patients in their $50 \mathrm{~s}$ and $60 \mathrm{~s}$ and rarely occurred in other age groups (Fig. 1g).

To identify the risk factors for severe ATE and ATEassociated death, the patients' characteristics, therapy for IBD, and laboratory data before the onset of ATE were compared between the groups with and without severe ATE and ATE-associated death. In the univariate and multivariate analyses, the only risk factor for severe ATE and ATE-associated death was the site of development of ATE, i.e., IHD (77.2\% in IHD vs. $23.1 \%$ at other sites; odds ratio [OR]: $66.7,95 \%$ confidence interval [CI]: 5.37-793.7, $p=0.0010$ ), as summarized in Table $3 \mathrm{a}$ and 3b. All variables included in the univariate analysis are described in Table S1.

Therapy for ATE consisted of antithrombotic therapy in 31 patients $(64.5 \%)$, fibrinolytic therapy in $8(16.7 \%)$, catheter intervention in $12(25.0 \%)$, and surgery in 4 $(8.3 \%)$; none received observation without therapy. The 
Table 1 Number and incidence of IBD patients with TE and severe TE and TE-associated death in the first nationwide surveillance

\begin{tabular}{|c|c|c|c|}
\hline & IBD total & $\mathrm{UC}$ & $\mathrm{CD}$ \\
\hline Number of total patients & 31,940 & 21,186 & 10,754 \\
\hline Number of patients developing TE $(\mathrm{N}, \%)$ & $\begin{array}{l}604 \\
(1.89 \%)\end{array}$ & $\begin{array}{l}374 \\
(1.77 \%)\end{array}$ & $\begin{array}{l}195 \\
(1.81 \%)\end{array}$ \\
\hline Number of patients developing ATE $(\mathrm{N}, \%)$ & $\begin{array}{l}278 \\
(0.87 \%)\end{array}$ & $\begin{array}{l}175 \\
(0.83 \%)\end{array}$ & $\begin{array}{l}103 \\
(0.96 \%)\end{array}$ \\
\hline Number of patients occurring VTE $(\mathrm{N}, \%)$ & $\begin{array}{l}328 \\
(1.03 \%)\end{array}$ & $\begin{array}{l}202 \\
(0.95 \%)\end{array}$ & $\begin{array}{l}91 \\
(0.84 \%)\end{array}$ \\
\hline Number of patients with severe TE and/or TE-associated death & $\begin{array}{l}65 \\
(0.20 \% *) \\
(10.7 \% * *)\end{array}$ & - & - \\
\hline Number of patients with TE-associated death & $\begin{array}{l}6 \\
(0.019 \% *) \\
(1.0 \% * *)\end{array}$ & $\begin{array}{l}6 \\
(0.028 \% *) \\
(1.6 \% * *)\end{array}$ & 0 \\
\hline Number of patients with ATE-associated death & $\begin{array}{l}2 \\
(0.0063 \% *) \\
(0.72 \% * *)\end{array}$ & $\begin{array}{l}2 \\
(0.0094 \% *) \\
(1.14 \% * *)\end{array}$ & 0 \\
\hline Number of patients with VTE-associated death & $\begin{array}{l}4 \\
(0.012 \% *) \\
(1.22 \% * *)\end{array}$ & $\begin{array}{l}4 \\
(0.019 \% *) \\
(1.98 \% * *)\end{array}$ & 0 \\
\hline $\begin{array}{l}\text { Estimated annual TE-associated mortality } \\
\text { (persons per 100,000 IBD patient-year) }\end{array}$ & 1.88 & 2.83 & 0 \\
\hline
\end{tabular}

proportion of patients who received antithrombotic and fibrinolytic therapy did not significantly differ between the groups with and without severe ATE and ATE-associated death. Hemorrhagic complications due to antithrombotic or fibrinolytic therapy arose in two cases $(4.2 \%)$, both in the group with severe ATE and ATE-associated death (Table S3).

The outcomes after ATE are described in Table S4. The average duration until a severe condition developed from the onset of ATE was 1.1 days. The duration until death from the onset of ATE was 0 and 320 days in the two cases that died. The outcomes after ATE consisted of improvement in $29(60.4 \%)$, retention of sequelae in $10(20.8 \%)$, death in $2(4.2 \%)$, and uncertain in 7 (14.6\%). The improvement rate in the group with severe ATE and ATEassociated death $(40.9 \%)$ tended to be lower than in the group without it $(76.9 \%)$, while the retention rate of sequelae $(31.8 \%)$ in the group with severe ATE and ATEassociated death tended to be higher than in the group without it (11.5\%).

\section{VTE}

A total of 167 IBD patients who developed VTE were examined. The demographics of the patients with IBD are shown in Table 2. The average of age at the onset of ATE was 47.3 years old, and the Montreal classification was A1 in $6(3.6 \%)$, A2 in $54(32.3 \%)$, and A3 in $107(64.7 \%)$. The proportion of males $(n=88,52.7 \%)$ was comparable to that of females $(n=79,47.3 \%)$. The average BMI was $20.6 \mathrm{~kg} / \mathrm{m}^{2}$. The proportion of UC cases $(n=120,71.2 \%)$ tended to be higher than that of CD ( $n=47,28.8 \%)$, and pancolitis-type (E3) UC accounted for $77.5 \%$ of all UC cases, whereas L3 of CD accounted for $70.2 \%$ of all CD cases. The average disease duration until the development of VTE was 117.1 months. The proportion of patients who were current or previous smokers was $28.8 \%$ and $7.2 \%$, respectively. Regarding comorbidities, $7.8 \%$ of patients ( $n=13)$ had malignancy, $4.7 \%(n=8)$ had DM, $7.8 \%$ $(n=13)$ had HT, $3.0 \%(n=5)$ had DLp, $3.0 \%(n=5)$ had renal dysfunction, $4.8 \%(n=8)$ had cardiovascular disease, $1.8 \%(n=3)$ had neuromuscular disease, and $2.4 \%(n=4)$ had a thrombotic tendency.

The proportion of patients with VTE who were hospitalized ( $n=120,71.9 \%$ ) was higher than that of patients 
Table 2 Characteristics of IBD patients who developed ATE and VTE

$$
\text { ATE } N=48
$$$$
56.3 \pm 15.1
$$

$1 / 7 / 40$

$33 / 15$

$162.2 \pm 13.8$

$59.2 \pm 13.6$

$22.1 \pm 3.6$

$41(85.4 \%)$

$7(14.6 \%)$

$2 / 9 / 30$

Type of UC (E1/E2/E3)

Type of CD (L1/L2/L3)

Disease duration until TE (months)

$\operatorname{EIM}(N, \%)$

Lifestyle habit and comorbidity at the onset of TE

History of smoking $(N, \%)$

History of alcohol drinking $(N, \%)$

History of VTE $(N, \%)$

Comorbidity

Malignancy

$\mathrm{DM}$

HT

DLp

Renal dysfunction

Cardiovascular disease

Cerebrovascular disease

Neuromuscular disease

Disease with thrombotic tendency

Detailed situation at onset of TE

Medical situation at onset of TE

Ambulatory $(N, \%)$

Hospitalization $(N, \%)$

Hospitalization until onset of TE (days)

Clinical activity at onset of TE

Partial Mayo (UC)

CDAI (CD)

Patients with moderate to severe IBD activity $(N, \%)$

Antithrombotic drugs before onset of TE $(N, \%)$

Concomitant treatment for IBD

5-ASA $(N, \%)$

Corticosteroid $(N, \%)$

Immunomodulator $(N, \%)$

Calcineurin inhibitor $(N, \%)$

Anti-TNF antibody $(N, \%)$

Anti-IL12/23 antibody $(N, \%)$

JAK inhibitor $(N, \%)$

Vedolizumab $(N, \%)$
$4 / 2 / 1$

$124.2 \pm 160.2$

$1(2.1 \%)$

$22(45.8 \%)$

$14(29.2 \%)$

$8(16.7 \%)$

$30(62.5 \%)$

$1(2.1 \%)$

$11(22.9 \%)$

$13(27.1 \%)$

$8(16.7 \%)$

$4(8.3 \%)$

$8(16.7 \%)$

$2(4.2 \%)$

$0(0 \%)$

$0(0 \%)$

$37(77.1 \%)$

$11(27.9 \%)$

$21.0 \pm 19.6$

$2.7 \pm 2.4$

$4(8.3 \%)$

$6(12.5 \%)$

$34(70.8 \%)$

$12(25.0 \%)$

$13(27.1 \%)$

$1(2.1 \%)$

$5(10.4 \%)$

$0(0 \%)$

$2(4.2 \%)$

$1(2.1 \%)$
$47(28.1 \%)$

VTE $N=167$

$47.3 \pm 18.4$

$6 / 54 / 107$

$88 / 79$

$164.4 \pm 8.5$

$55.8 \pm 12.2$

$20.6 \pm 4.06$

$120(71.2 \%)$

$47(28.8 \%)$

$4 / 19 / 94$

$11 / 2 / 33$

$117.1 \pm 128.2$

$22(13.2 \%)$

$45(26.9 \%)$

$35(21.9 \%)$

$12(7.2 \%)$

$73(43.7 \%)$

$13(7.8 \%)$

$8(4.7 \%)$

$13(7.8 \%)$

$5(3.0 \%)$

$5(3.0 \%)$

$8(4.8 \%)$

$0(0 \%)$

$3(1.8 \%)$

$4(2.4 \%)$

$120(71.9 \%)$

$20.3 \pm 20.8$

$4.6 \pm 2.9$

$239.3 \pm 79.1$

$55(32.9 \%)$

$3(1.8 \%)$

$109(65.3 \%)$

$88(52.7 \%)$

$33(19.8 \%)$

$10(6.0 \%)$

$28(16.7 \%)$

$1(0.6 \%)$

$1(0.6 \%)$

$0(0 \%)$ 
Table 2 continued

\begin{tabular}{|c|c|c|}
\hline & ATE $N=48$ & VTE $N=167$ \\
\hline Cytapheresis $(N, \%)$ & $1(2.1 \%)$ & $16(9.6 \%)$ \\
\hline Central venous catheter $(N, \%)$ & $3(6.3 \%)$ & $80(48.0 \%)$ \\
\hline Bowel resection $(N, \%)$ & $4(8.3 \%)$ & $52(31.3 \%)$ \\
\hline \multicolumn{3}{|l|}{ Laboratory data before onset of $\mathrm{TE}$} \\
\hline $\mathrm{WBC}(/ \mu \mathrm{l})$ & $7321 \pm 3679$ & $8112 \pm 4273$ \\
\hline $\mathrm{Hb}(\mathrm{g} / \mathrm{dl})$ & $12.2 \pm 2.1$ & $11.2 \pm 2.2$ \\
\hline Ht $(\%)$ & $37.4 \pm 6.2$ & $34.2 \pm 6.4$ \\
\hline Plt $\left(\times 10^{6} / \mu \mathrm{l}\right)$ & $48.4 \pm 90.3$ & $32.0 \pm 15.8$ \\
\hline $\mathrm{T}-\mathrm{P}$ (g/dl) & $6.5 \pm 1.0$ & $6.8 \pm 5.4$ \\
\hline $\mathrm{Alb}(\mathrm{g} / \mathrm{dl})$ & $3.5 \pm 0.8$ & $3.1 \pm 0.8$ \\
\hline BUN mg/dl) & $14.9 \pm 5.3$ & $11.6 \pm 6.3$ \\
\hline Cre $(\mathrm{mg} / \mathrm{dl})$ & $0.95 \pm 0.80$ & $0.82 \pm 0.87$ \\
\hline CRP (mg/dl) & $2.53 \pm 4.0$ & $3.09 \pm 4.4$ \\
\hline ESR (mm/hour) & $38.3 \pm 24.6$ & $36.8 \pm 26.1$ \\
\hline PT-INR & $1.12 \pm 0.19$ & $1.42 \pm 2.88$ \\
\hline APTT (sec) & $34.6 \pm 9.3$ & $37.6 \pm 56.6$ \\
\hline Fib (mg/dl) & $358 \pm 110$ & $371.8 \pm 113.4$ \\
\hline D-dimer (ng/dl) & $10.5 \pm 28.3$ & $7.9 \pm 21.3$ \\
\hline FDP (ng/dl) & $52.5 \pm 98.8$ & $24.6 \pm 52.5$ \\
\hline AT-III (\%) & $91.8 \pm 20.8$ & $90.4 \pm 20.1$ \\
\hline
\end{tabular}

$B W$ body weight, $B M I$ body mass index, $I B D$ inflammatory bowel disease, $A T E$ arterial thromboembolism VTE venous thromboembolism, $U C$ ulcerative colitis, $C D$ Crohn's disease, EIM extra-intestinal manifestation, $D M$ diabetes mellitus, $H T$ hypertension, $D L p$ dyslipidemia, 5-ASA 5-amynosalicylate, $T N F$ tumor necrosis factor, $I L$ interleukin, JAK Janus kinase, $W B C$ White blood cell count, $H b$ hemoglobin, $H t$ hematocrit, $P l t$ platelet, $T-P$ total protein, $A l b$ albumin, $B U N$ blood urea nitrogen, $C r e$ creatinine, CRP C-reactive protein, ESR erythrocyte sedimentation rate, $P T$-INR prothrombin international rate, APTT activated partial thrombin time, Fib fibrinogen, FDP fibrin degradation product, AT-III antithrombin-III

who were ambulatory $(n=47,28.1 \%)$, which was the opposite of the tendency seen in cases with ATE. The average hospitalization period in patients who developed VTE was 20.3 days. The average pMayo score and CDAI in patients with UC and CD were 4.6 and 239.3, respectively, and the proportion of patients with moderate to severe clinical activity was $32.9 \%(n=55)$, suggesting that the clinical activity in patients with VTE tended to be higher than in those with ATE. The treatment used for IBD was 5-ASA in 109 patients $(65.3 \%)$, corticosteroids in 88 $(52.7 \%)$, IM in $33(19.8 \%)$, CNI in $10(6.0 \%)$, anti-TNF antibody in $28(16.7 \%)$, anti-IL12/23 antibody in $1(0.6 \%)$, JAK inhibitor in $1(0.6 \%)$, plasmacytapheresis in 16 $(9.6 \%), \mathrm{CVC}$ in $80(48.0 \%)$, and bowel resection in 52 $(31.3 \%)$; VDZ was not used by any patient. Of note, the proportion of VTE patients treated with corticosteroids, $\mathrm{CVC}$, and bowel resection tended to be higher than that of ATE patients (vs. 25.0\%, 6.3\%, and 8.3\%, respectively).

Laboratory data before the onset of VTE are shown in Table 2. The number of VTE instances at each site among the 167 cases is shown in Fig. 2a. These patients had DVT in $\mathrm{L}(n=45,26.9 \%)$, PA $(n=17,10.2 \%)$, DVT in L and
PA $(n=26,15.6 \%), \operatorname{PMV}(n=31,18.6 \%), \operatorname{CVS}(n=8$, $4.8 \%)$, and CR $(n=40,24.0 \%)$. The proportion of VTEassociated severity and death at each site is shown in Fig. 2b. The proportion of patients who developed severe VTE and VTE-associated death was $17.0 \%(n=32$; death: $2.4 \%$ ) in the cohort of the secondary surveillance. The proportion of severe VTE and VTE-associated death was $2.0 \%(n=1)$ in DVT in L, $36.0 \%(n=6)$ in PA, $38.0 \%$ $(n=10)$ in DVT in L and PA, 26.0\% $(n=8)$ in PMV, $63.0 \%(n=5)$ in CVS, and $5.0 \%(n=2)$ in CR. The proportions of severe VTE and VTE-associated death in L + PA (38.0\%) and CVS (63.0\%) were significantly higher than those of L (2.0\%) and CR $(5.0 \%)(p=0.003$ in $\mathrm{L}+\mathrm{PA}$ vs. $\mathrm{L}, p=0.027$ in $\mathrm{L}+\mathrm{PA}$ vs. $\mathrm{CR}, p=0.0002$ in CVS vs. L, $p=0.004$ in CVS vs. CR). The proportion of severe VTE and VTE-associated death in PA (36\%) was significantly higher than that in $\mathrm{L}(p=0.019)$, and that in PMV (26.0\%) tended to be higher than that in $\mathrm{L}$ $(p=0.085)$. The mortality rate due to VTE was $18.0 \%$ $(n=3)$ and $13.0 \%(n=3)$ in PA and CVS, respectively, and no deaths occurred in cases concerning $\mathrm{L}, \mathrm{L}+\mathrm{PA}$, PMV, PMV, and CR. The proportion of severe VTE and 
(a)

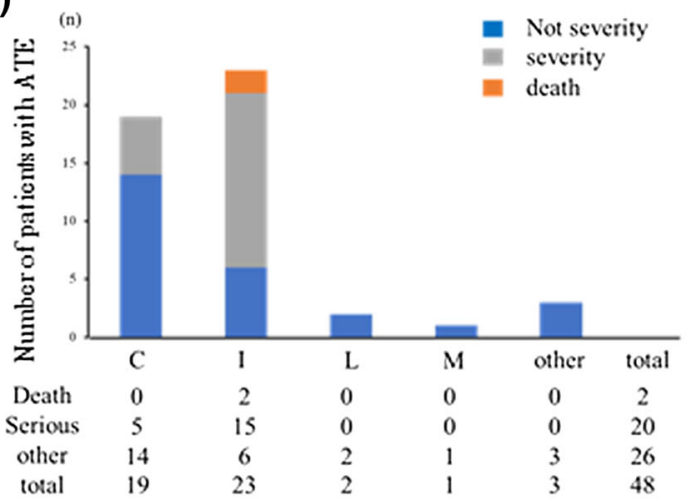

C: cerebrovascular disease

I: ischemic heart disease

L: limbs and peripheral arterial thromboembolism

M:mesenteric arterial thromboembolism

(c)
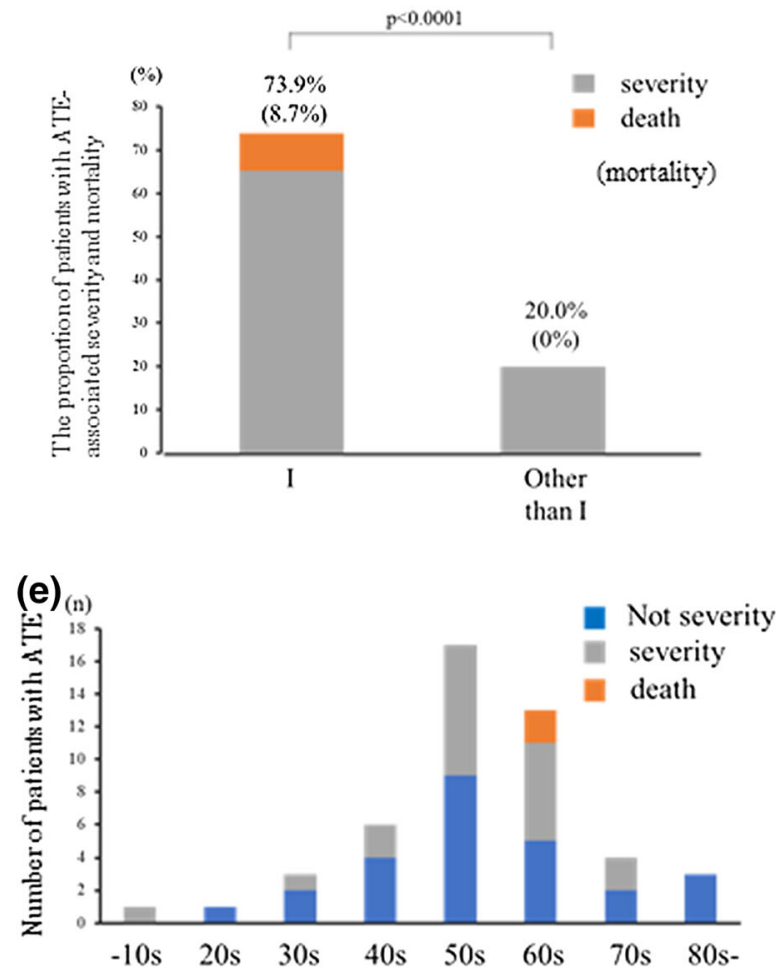

$(g)_{(\mathrm{n})}$

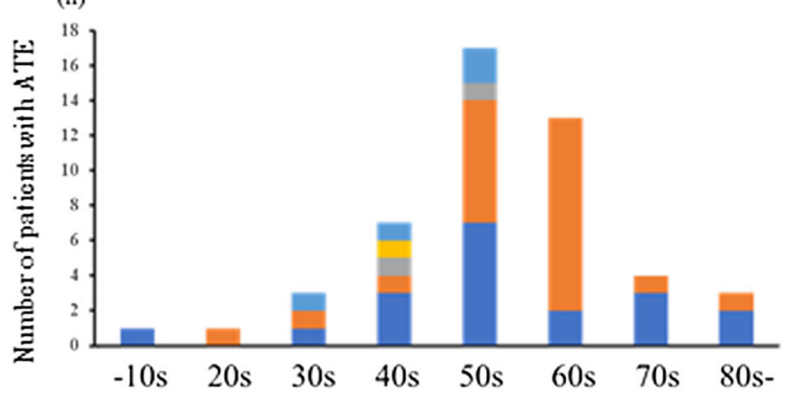

(b)

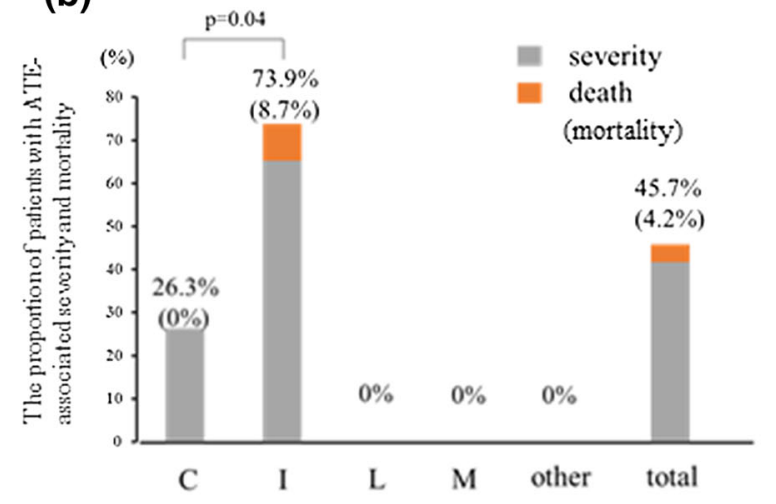

(d)
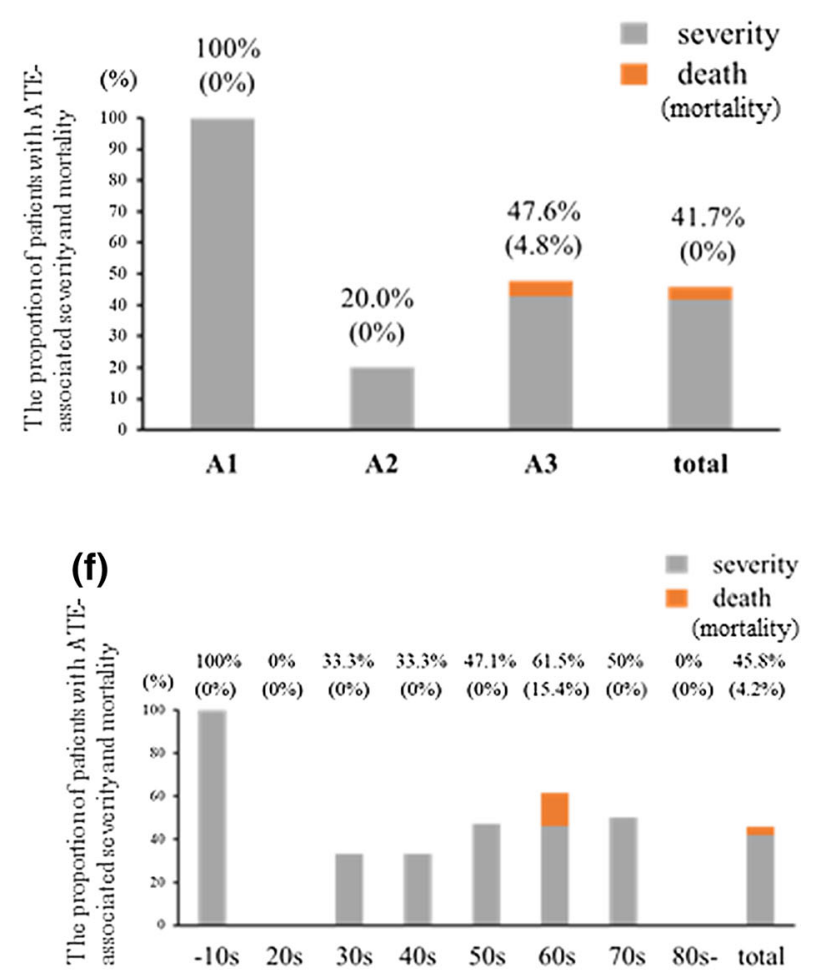

- C(Cercbrovascular discase)

- I(Ischemic heart disease)

L Llimbs artery)

- M(mesenteric artery)

- O(other) 
4Fig. 1 The incidence of severe ATE and ATE-associated death in IBD patients. Each figure shows a the number of patients with severe ATE and ATE-associated death at each site, $\mathbf{b}$ the proportion of severe ATE and ATE-associated death at each site and $\mathbf{c}$ when classified as IHD versus others, $\mathbf{d}$ the proportion of severe ATE and ATE-associated death when classified into three groups based on the Montreal classification, e the number of cases of severe ATE and ATE-associated death by age (every decade), $\mathbf{f}$ the proportion of severe ATE and ATE-associated death by age (every decade), and $\mathbf{g}$ the incidence of ATE at each site and for each age group

VTE-associated death in PA/PM/CVS (34.1\%; mortality, $4.9 \%)$ was much higher than that at other sites $(4.7 \%)$ $(p<0.0001)$ (Fig. 2c). The proportion of severe VTE and VTE-associated death in patients $\leq 45$ years old $(28.0 \%)$ was significantly higher than that in patients $\geq 46$ years old $(10.6 \%, p=0.007)$. Notably, death due to VTE occurred only in patients $\leq 45$ years old (mortality: $4.9 \%$ ) (Fig. 2d). Regarding the Montreal classification, the proportions of severe VTE and VTE-associated death of $50.0 \%(n=3), 25.9 \%(n=14)$, and $13.9 \%(n=15)$ in A1, $\mathrm{A} 2$, and A3, respectively, showed no significant difference, although the proportions gradually increased with decreasing age (Fig. 2e). Furthermore, on analyzing the age groups by decade, the incidence of VTE peaked in patients in their $40 \mathrm{~s}$ among all generations. The proportion of severe VTE and VTE-associated death in patients in their $\leq 10 \mathrm{~s}, 20 \mathrm{~s}, 30 \mathrm{~s}, 40 \mathrm{~s}, 50 \mathrm{~s}, 60 \mathrm{~s}, 70 \mathrm{~s}$ and $\geq 80 \mathrm{~s}$ was $35.7 \%$ (5/14), $23.4 \%$ (4/17), $28.6 \%$ (8/28), $24.2 \%$ (8/ $33), 10.3 \%$ (3/29), $4.3 \%$ (1/23), $11.1 \%$ (2/18), and $20.0 \%$ $(1 / 5)$, respectively. The proportion of severe VTE and VTE-associated death in patients in their $10 \mathrm{~s}$ to $40 \mathrm{~s}$ tended to be higher than that in patients in their $50 \mathrm{~s}$ to $70 \mathrm{~s}$, while the incidence of VTE in patients in their $50 \mathrm{~s}$ to $70 \mathrm{~s}$ was comparable to that in patients in their $10 \mathrm{~s}$ to $40 \mathrm{~s}$ (Fig. 2f, g). Regarding the development site and age, CVS predominantly occurred in patients in their $10 \mathrm{~s}$ to $40 \mathrm{~s}$, while the incidence of $\mathrm{L}$ and/or PA gradually increased with age (Fig. 2h).

To identify the risk factors for severe VTE and VTEassociated death, the patients' characteristics, therapy for IBD, and laboratory data before the onset of VTE were compared between the groups with and without severe VTE and VTE-associated death. In the univariate analysis, the risk factors for severe VTE and VTE-associated death were a young age ( $\leq 45$ years old), no history of smoking, site of development of VTE (PA, PMV, CVS), moderate to severe disease activity, non-use of anti-TNF antibody, and an elevated CRP level before the onset of VTE ( $\geq 1.5 \mathrm{mg}$ / dL) (Table 4a). In the multivariate analysis, the independent risk factors for severe VTE and VTE-associated death were age $\leq 45$ years old (OR: $2.84,95 \%$ CI: $1.05-7.69$, $p=0.038$ ), the site of development of VTE (PA, PMV and
CVS) (OR: 9.79, 95\% CI: 3.03-31.6, $p=0.00013$ ), and disease severity (moderate to severe) (OR: $2.79,95 \% \mathrm{CI}$ : 1.04-7.47, $p=0.0415$ ) (Table 4b). All variables included in the univariate analysis are described in Table S2.

Therapy for VTE consisted of antithrombotic therapy in 140 patients $(83.8 \%)$, fibrinolytic therapy in $13(7.8 \%)$, IVC filter in $11(6.6 \%)$, catheter intervention (other than an IVC filter) in $9(5.4 \%)$, surgery in $8(4.8 \%)$, and observation without therapy in $20(12.0 \%)$. Fibrinolytic therapy in the group with severe VTE and VTE-associated death $(n=6,18.8 \%)$ was performed more frequently than in the group without it $(n=7,5.2 \%)$, and there was no untreated case in the group of severe VTE and VTE-associated death. In addition, $15.0 \%$ of patients without severe VTE and VTE-associated death were untreated. A significant difference was observed in the incidence of untreated cases between the two groups $(p=0.015)$ (Table S5).

Hemorrhagic complications due to antithrombotic or fibrinolytic therapy arose in nine cases (5.4\%), and the proportion of hemorrhagic complications in the group with severe VTE and VTE-associated death (16.1\%) was significantly higher than in the group without it (3.0\%) $(p=0.012)$ (Table S5).

The outcomes after VTE are described in Table 5. The average duration until a severe condition developed from the onset of VTE was 3.3 days. The average duration until death from the onset of VTE was 13.5 days. VTE was resolved and remained in $95(56.9 \%)$ and $52(31.4 \%)$ patients, respectively, sequelae remained in $6(3.6 \%)$ patients, 4 (2.4\%) patients died, while the outcomes in 10 $(6.0 \%)$ patients were uncertain. In the group with severe VTE and VTE-associated death, VTE was resolved and sequelae remained in nine $(40.9 \%)$ and seven $(31.8 \%)$ patients, respectively. The proportion with sequelae in the group of severe VTE and VTE-associated death (12.5\%) tended to be higher than in the group without severe VTE and VTE-associated death (0\%), while the proportions with improvement regardless of the elimination of VTE were comparable between the groups ( $75.0 \%$ vs. $85.9 \%)$.

\section{Discussion}

To the best of our knowledge, the present study is the first nationwide cohort study to describe the incidence and risk factors of severe TE and TE-associated death in IBD patients in Japan and the largest such study in Asia (questionnaire based on patients' background characteristics). In the first large cohort surveillance (including over 30,000 IBD patients across 10 years), the incidence of TE in IBD patients was $1.89 \%$ (188.8 per 100,000 IBD personyears). In addition, the incidence of TE between patients with UC (1.77\%, 176.5 per 100,000 UC person-years) and 
Table 3 Univariate and multivariate analyses comparing the characteristics between groups with and without severe ATE and ATE-associated death.

\begin{tabular}{|c|c|c|c|}
\hline Univariate analysis & Severity and death $(n=22)$ & Other $(n=26)$ & $p$ value \\
\hline Age at the onset (years) & $55.6 \pm 15.5$ & $57.0 \pm 15.4$ & 0.757 \\
\hline Male $(N, \%)$ & $17(19.3 \%)$ & $15(19.0 \%)$ & 1 \\
\hline BW (kg) & $55.9 \pm 14.3$ & $62.1 \pm 12.6$ & 0.119 \\
\hline BMI (kg/m2) & $21.7 \pm 3.5$ & $22.5 \pm 3.6$ & 0.439 \\
\hline Type of IBD & & & 1 \\
\hline $\mathrm{UC}(N, \%)$ & $19(86.4 \%)$ & $22(79.2 \%)$ & \\
\hline $\mathrm{CD}(N, \%)$ & $3(13.6 \%)$ & $4(84.6 \%)$ & \\
\hline Disease duration until ATE (months) & $81.7 \pm 108.3$ & $157.5 \pm 186.9$ & 0.135 \\
\hline History of smoking $(N, \%)$ & $11(50.0 \%)$ & $11(42.3 \%)$ & 0.772 \\
\hline Comorbidity & $14(63.6 \%)$ & $16(61.5 \%)$ & 1 \\
\hline Malignancy & $0(0 \%)$ & $1(3.8 \%)$ & 1 \\
\hline $\mathrm{DM}$ & $3(13.6 \%)$ & $8(30.8 \%)$ & 0.189 \\
\hline HT & $5(22.7 \%)$ & $8(30.7 \%)$ & 0.746 \\
\hline DLp & $4(18.1 \%)$ & $4(15.4 \%)$ & 1 \\
\hline Renal dysfunction & $0(0 \%)$ & $4(15.4 \%)$ & 0.114 \\
\hline Cardiovascular disease & $4(18.1 \%)$ & $4(15.4 \%)$ & 1 \\
\hline Cerebrovascular disease & $2(9.1 \%)$ & $0(0 \%)$ & 0.205 \\
\hline Type of ATE CVD/IHD/L/M/other & $5 / 17 / 0 / 0 / 0$ & $14 / 6 / 2 / 1 / 3$ & 0.0041 \\
\hline Proportion of IHD & $17(77.2 \%)$ & $6(23.1 \%)$ & 0.00038 \\
\hline Hospitalization at onset of ATE & $5(22.7 \%)$ & $17(23.1 \%)$ & 1 \\
\hline Hospitalization until onset of ATE (days) & $28.8 \pm 27.5$ & $14.8 \pm 9.6$ & 0.320 \\
\hline \multicolumn{4}{|l|}{ Clinical activity at onset of ATE } \\
\hline Partial Mayo (UC) & $2.5 \pm 2.2$ & $2.2 \pm 1.9$ & 0.698 \\
\hline Patients with moderate to severe IBD activity $(N, \%)$ & $3(13.6 \%)$ & $1(3.8 \%)$ & 0.320 \\
\hline Antithrombotic drugs before onset of $\operatorname{ATE}(N, \%)$ & $4(18.2 \%)$ & $2(7.7 \%)$ & 0.484 \\
\hline \multicolumn{4}{|l|}{ Concomitant usage of treatment for IBD } \\
\hline Corticosteroid $(N, \%)$ & $4(18.2 \%)$ & $8(30.8 \%)$ & 0.495 \\
\hline Immunomodulator $(N, \%)$ & $5(30.7 \%)$ & $8(30.8 \%)$ & 0.739 \\
\hline Anti-TNF antibody $(N, \%)$ & $2(9.1 \%)$ & $3(11.5 \%)$ & 1 \\
\hline Central venous catheter $(N, \%)$ & $3(13.6 \%)$ & $0(0 \%)$ & 0.084 \\
\hline Bowel resection $(N, \%)$ & $1(4.5 \%)$ & $3(11.5 \%)$ & 0.613 \\
\hline \multicolumn{4}{|l|}{ Laboratory data before onset of ATE } \\
\hline $\mathrm{WBC}(/ \mu \mathrm{l})$ & $7516 \pm 3937$ & $7196 \pm 3595$ & 0.804 \\
\hline $\mathrm{Hb}(\mathrm{g} / \mathrm{dl})$ & $12.7 \pm 1.7$ & $12.0 \pm 2.4$ & 0.391 \\
\hline Plt $\left(\times 10^{6} / \mu \mathrm{l}\right)$ & $37.8 \pm 60.5$ & $55.2 \pm 105.9$ & 0.578 \\
\hline Alb (g/dl) & $3.7 \pm 0.68$ & $3.4 \pm 0.89$ & 0.367 \\
\hline Cre $(\mathrm{mg} / \mathrm{dl})$ & $0.77 \pm 0.21$ & $1.06 \pm 0.99$ & 0.286 \\
\hline CRP (mg/dl) & $2.97 \pm 4.7$ & $2.23 \pm 3.5$ & 0.597 \\
\hline D-dimer (ng/dl) & $17.1 \pm 38.6$ & $2.65 \pm 3.9$ & 0.430 \\
\hline FDP (ng/dl) & $62.6 \pm 111.1$ & $12.0 \pm 23.5$ & 0.711 \\
\hline AT-III (\%) & $95.5 \pm 22.1$ & $77.0 \pm 25.4$ & 0.508 \\
\hline \multicolumn{4}{|l|}{ Multivariate analysis } \\
\hline Variables & OR $(95 \% \mathrm{CI})$ & & $p$ value \\
\hline $\mathrm{BW}$ & $0.99(0.86-1.12)$ & & 0.824 \\
\hline Comorbidity-DM & $5.08(0.38-66.2)$ & & 0.21 \\
\hline Comorbidity-Renal dysfunction & & & 0.997 \\
\hline
\end{tabular}


Table 3 continued

\begin{tabular}{lll}
\hline $\begin{array}{l}\text { Multivariate analysis } \\
\text { Variables }\end{array}$ & OR (95\% CI) & $p$ value \\
\hline Site of ATE: IHD & $66.7(5.37-793.7)$ & 0.0010 \\
Central venous catheter & & 0.997
\end{tabular}

$B W$ body weight, $B M I$ body mass index, $I B D$ inflammatory bowel disease, $V T E$ venous thromboembolism, $U C$ ulcerative colitis, $C D$ Crohn's disease, $D M$ diabetes mellitus, $H T$ hypertension, $D L p$ dyslipidemia, $C V D$ cerebrovascular disease, $I H D$ ischemic heart disease, $L$ limbs and peripheral arterial thromboembolism, $M$ mesenteric arterial thromboembolism, 5-ASA: 5-amynosalicylate, $T N F$ tumor necrosis factor, $W B C$ White blood cell count, $H b$ hemoglobin, Plt platelet, $A l b$ albumin, $B U N$ blood urea nitrogen, $C r e$ creatinine, $C R P$ C-reactive protein, $F D P$ fibrin degradation product, $A T$-III antithrombin-III, $C I$ confidence interval, $O R$ odds ratio

CD (1.81\%, 181.3 per person-year) was comparable. Regarding ATE in the present study, the incidence and mortality rate in Japanese IBD patients was $0.87 \%(86.9$ per 100,000 IBD person-years) and $4.2 \%$ per IBD-associated ATE patients. The age-standardized incidence rate of stroke and coronary artery disease in the Japanese general population (1988-2000) was reported to be 1,446 per 100,000 person-years in a large cohort study [25], suggesting that Japanese IBD patients are unlikely to have an increased risk of ATE compared to non-IBD patients. In contrast, according to a meta-analysis by Sighn et al., the incidence of ATE (including all IHD, CVD, and peripheral ATE) in Western IBD patients was $5.92 \%$ (9,050 of 152,756 IBD patients) ${ }^{11}$, which was higher than in our study. Several recent reports have suggested that IBD patients in Western countries had a 1.18- to 8.07-fold higher risk of ATE, including IHD and CVD, than non-IBD patients [9-11]. These findings suggest that the incidence of IBD-associated ATE markedly differs between Asian and Western countries. The low incidence and mortality rate of ATE in Asian IBD patients might be associated with the different incidence of ATE in the general population between Asian and Western countries [26]. Regarding VTE in the present study, the incidence in IBD was $1.03 \%$ (102.5 per 100,000 IBD person-years) in Japan. Weng et al. reported that the incidence of VTE was $0.9 \%$ in the hospitalized cohort and 115 per 100,000 person-years in an East Asian cohort and that the incidence was comparable between UC and CD [20]. This showed that the incidence of VTE was almost 100 per 100,000 IBD person-years, which was much higher than that in the general Asian population $[27,28]$. In Western countries, the incidence of VTE was reported to be 74.5 to 314 per 100,000 personyears (14 to 30 in DVT and 10 to 20 , and 13 to 31 and 10 to 11 in UC and CD patients, respectively) [4, 29-31]. Unlike ATE, the incidence of IBD-associated VTE in Asian countries [32-36] was not lower than that in Western countries. While ATE is known to develop in association with arterial sclerosis, VTE is thought to develop due to the excessive activation of inflammation- and coagulation- related molecules [37, 38]. The different pathogenesis might lead to the different incidences between ATE and VTE in Asian IBD patients.

On comparing the characteristics between the patients with ATE and VTE, regardless of TE-associated severity or death, VTE patients tended to develop at a younger age and have a lower BMI, lower rate of a smoking history and comorbidities (especially DM, HT, CVD), higher clinical activity and higher rates of hospitalization (average pMayo in UC $4.6 \pm 2.9$ vs. $2.7 \pm 2.4$ and the proportion of moderate to severe activity $32.9 \%$ vs. $8.3 \%$ ), concomitant use of corticosteroids, and a central venous catheter and bowel resection at the onset of TE than ATE patients (Table 2). These findings suggest that a higher disease activity and rate of thrombogenesis-related medical intervention (corticosteroid, catheter indwelling, and surgery) were potential risk factors in VTE with IBD, whereas common risk factors for TE, such as obesity, lifestyle, and comorbidities (DM, HT, and cardiovascular disease), might be more strongly associated with the onset of ATE with IBD. This discrepancy in the characteristics between ATE and VTE may have contributed to the differences in the incidences of ATE and VTE between IBD patients and the general population.

In this study, the incidence of severe TE and TE-associated death with IBD was $0.20 \%$ among all IBD patients and $10.7 \%$ among IBD patients with TE. The mortality of IBD patients with TE was $0.019 \%$ among all IBD patients (annual mortality due to TE: 1.88 per 100,000 personyears) and $1.0 \%$ among IBD patients with TE, which was not higher than that in general population. Regarding ATE, a meta-analysis in Western countries showed that the mortality of cardiovascular disease in IBD patients was not higher than that in the general population [8], although the risk of ATE in IBD patients has been reported to be higher than that in non-IBD controls [9-11]. These findings suggest that the incidences of severe ATE and ATE-associated death in IBD patients were not higher than those in the general population in Asian or Western countries. Regarding VTE in the present study, the mortality of VTE 
(a)

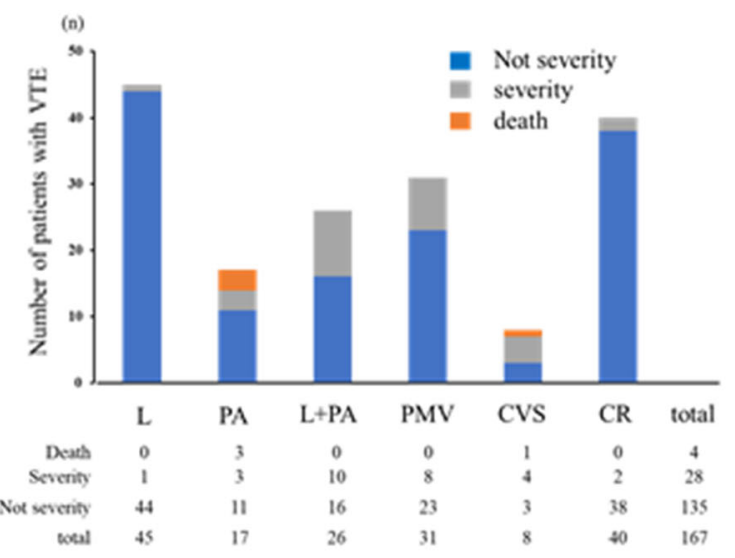

(c)

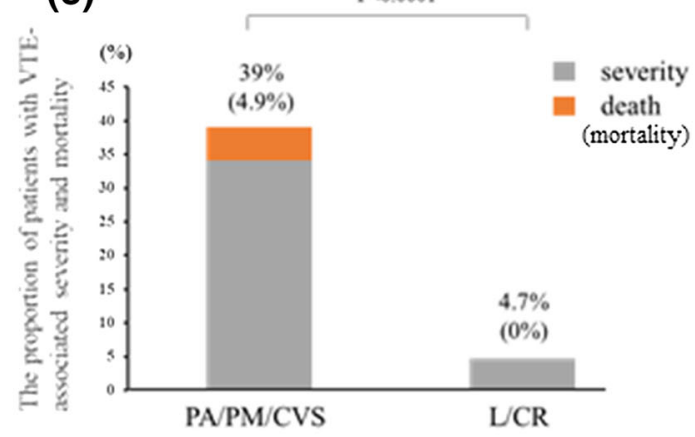

(e)

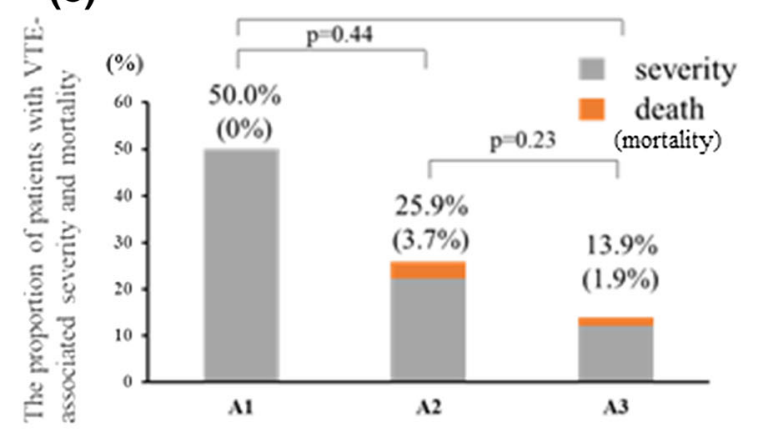

(g)

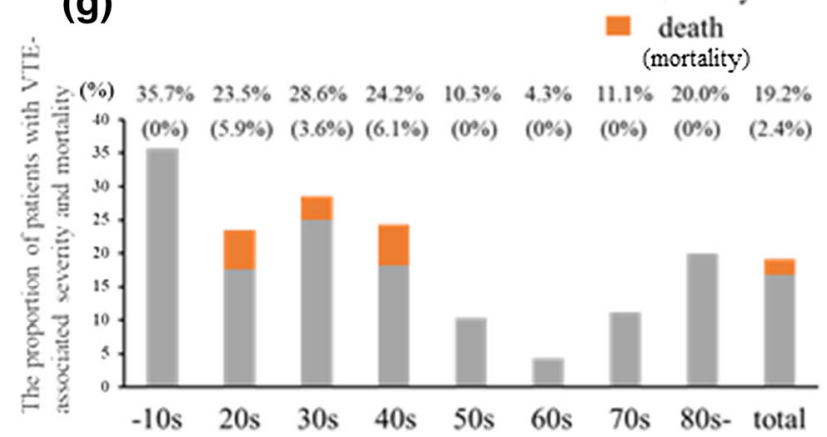

(b)

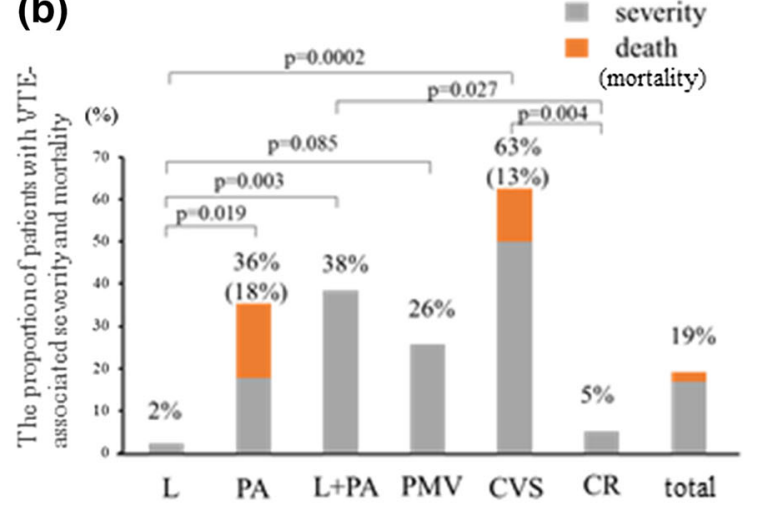

L: lower limbs PA: Pulmonary antery

PM: Portal and mesenteric vein

CVS: Cerebral venous sinus

CR: Catherer related

(d)

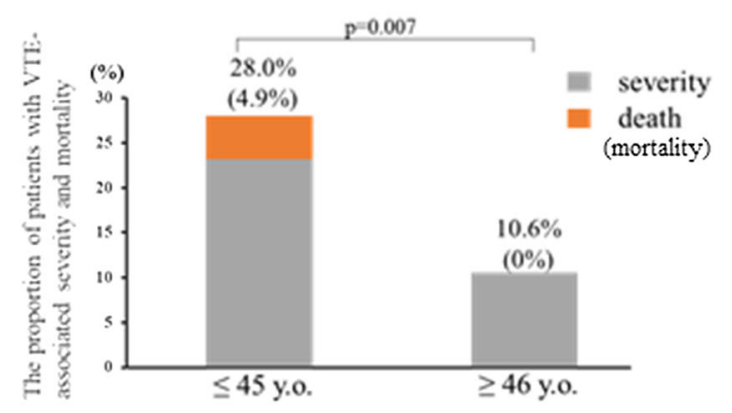

(f)
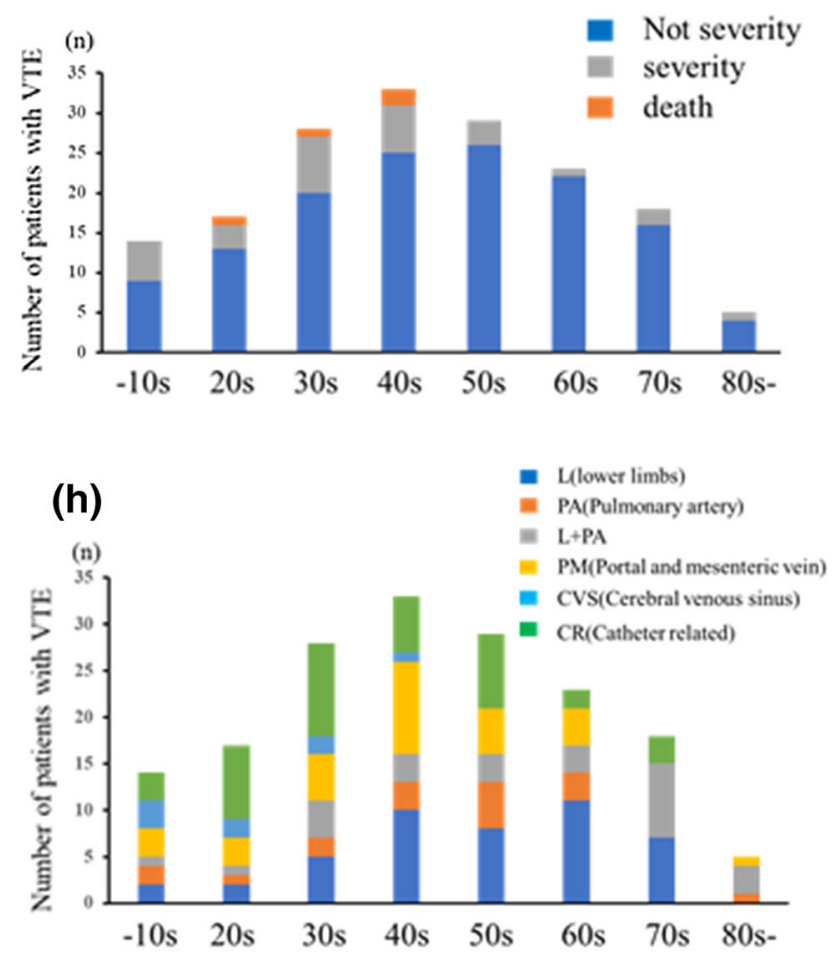
4 Fig. 2 The incidence of severe VTE and VTE-associated death in IBD patients. Each figure shows a the number of patients with severe VTE and VTE-associated death in each developing site, b the proportion of severe VTE and VTE-associated death in each developing site and c when classified with IHD and the others, d the proportion of severe VTE and VTE-associated death when classified with two groups in a boarder of age 45 and $\mathbf{e}$ three groups based on Montreal classification, $\mathbf{f}$ number of severe VTE and VTEassociated death in each age (every 10 years), $\mathbf{g}$ the proportion of severe VTE and VTE-associated death in each age (every 10 years), and $\mathbf{h}$ the incidence of VTE at each site and for each age group

with IBD was $0.012 \%$ among all IBD patients and $1.22 \%$ among IBD patients with VTE. In previous studies from Western and Latin American countries, VTE-associated mortality in IBD was reported to range from 10.7 to $25 \%$ [12-15]. Nguyen et al. reported that the risk of in-hospital mortality in IBD patients with VTE was 2.5- and 2.1-fold higher than that in IBD patients without VTE and non-IBD patients with VTE, respectively [15]. Recently, Weng et al. reported that the in-hospital mortality of VTE was $8.3 \%$ (2/ 24) among patients in an East Asian cohort based on a small sample size [20]. It is difficult to precisely determine the differences in mortality rates between Asian and Western countries due to the differences in study designs and sample sizes. Conversely, to our knowledge, no data on the incidence or outcomes of severe VTE have been published in Asian or Western countries. Our results indicate that the incidence of severe VTE was almost $20 \%$, with certain cases having higher risks of persistent VTE, sequelae, and death. This suggests that aggressive screening is needed to detect VTE before its severity progresses to improve the prognosis of IBD patients with VTE.

In cases of ATE, the proportion of severity and death among IHD patients was markedly higher than that among patients with CVD or other ATE. Our multivariate analysis revealed that IHD was the only independent factor associated with severe ATE and ATE-associated death in IBD patients. A recent study from Western countries showed that a higher disease activity carried a potential risk of ATE $[39,40]$; however, in our study, the clinical activity at the onset of ATE in the group with severe ATE and ATEassociated death and the average pMayo value of 2.5 were comparable to those in the group without severe ATE and ATE-associated death. The differences in the physical condition and prevalence of comorbidities (including traditional risk factors for CVD and IHD) between Asian and Western populations might have to be taken into consideration.

The multivariate analysis in this study found that the risk factors for severe VTE and VTE-associated death with IBD were a young age ( $\leq 45$ years old), the site of VTE (PA, PMV, and CVS), and the disease severity (moderate to severe). CVS is known to be associated with the highest risk of severity and death in young patients. Elevated proportions of pulmonary artery embolism and PMV have been demonstrated in young patients. These findings indicate the high risk of severity and death in young IBD patients with PA, PMV, and CVS, although the incidence of IBD-associated TE is low in the younger population. Our study also showed that a high disease activity was a positive risk factor, whereas the usage of anti-TNF antibody might be a negative one for severe VTE and VTEassociated death. A strong inflammatory condition is associated with hypercoagulation status due to increases in coagulation-associated mediators, including factors V, VII, and VIII, lipoprotein (a), and fibrinogen, and decreases in antithrombin III, protein $\mathrm{C} /$ protein $\mathrm{S}$, and tissue factor pathway inhibitor and the platelet activity [37]. TNF- $\alpha$ is known to induce the downregulation of endothelial protein C receptors on thin capillary endothelial cells [38]. A recent French cohort study also showed that the usage of anti-TNF antibody was associated with a reduction in the risk of acute arterial events [39]. These findings clearly indicate that the disease activity is closely associated with the risk of VTE in IBD patients. In this study, very few patients who were administered biologics other than antiTNF antibody were included, as the study period was set before 2017 when biologics other than anti-TNF antibody were not yet clinically available. Therefore, the efficacy of biologics other than anti-TNF antibody for reducing the rate of TE-associated severity and death was not examined in our study despite being expected due to the comparable clinical effectiveness to anti-TNF antibody. In the future, the effects of various agents, including antibody formulations and JAK inhibitors, on the incidence of TE and TEassociated severity and death should be evaluated.

Several limitations associated with this nationwide cohort study warrant mention. First, the demographics of the total IBD patients at the primary surveillance were lacking because this study aims to clarify the incidence and risk factors of severe TE and TE-associated death in IBD patients. Second, about $65 \%$ of the IBD patients with ATE and VTE who had been included in the primary surveillance were not included in the secondary surveillance. However, this study was the first of its kind to clarify the actual state of both ATE and VTE in an Asian population and is the largest nationwide cohort study conducted to date, including over 30,000 IBD patients from referral IBD centers and general hospitals in Japan. Third, this survey targeted high-volume institutions and referral centers for IBD participating in the Research Committee of Inflammatory Bowel Disease the Ministry of Health and Welfare of Japan. Therefore, the study population enrolled in our study might have been biased to some degree, as more refractory and intractable patients with IBD may have been 
Table 4 Univariate and multivariate analyses comparing the characteristics between groups with and without severe VTE and VTE-associated death

\begin{tabular}{|c|c|c|c|}
\hline \multirow[t]{2}{*}{ Univariate analysis } & \multirow[b]{2}{*}{ Severity and death $(n=32)$} & \multirow[b]{2}{*}{ Other $(n=135)$} & \multirow[b]{2}{*}{$p$ value } \\
\hline & & & \\
\hline Age at the onset (years) & $40.1 \pm 17.5$ & $49.1 \pm 18.2$ & 0.013 \\
\hline A1/A2/A3 & $3 / 14 / 15$ & $3 / 40 / 92$ & 0.030 \\
\hline Age $\leq 45$ years $(\mathrm{N}, \%)$ & $23(28.0 \%)$ & $9(10.6 \%)$ & 0.0056 \\
\hline Male $(\mathrm{N}, \%)$ & $17(19.3 \%)$ & $15(19.0 \%)$ & 1 \\
\hline BMI (kg/m2) & $21.5 \pm 4.7$ & $20.4 \pm 3.9$ & 0.180 \\
\hline Type of IBD & & & 0.513 \\
\hline $\mathrm{UC} / \mathrm{CD}(\mathrm{N}, \%)$ & $25(20.8 \%) / 7(14.9 \%)$ & $95(79.2 \%) / 40(85.1 \%)$ & \\
\hline Type of UC (E1/E2/E3) & $3 / 3 / 19$ & $1 / 16 / 75$ & 0.182 \\
\hline Type of CD (L1/L2/L3) & $2 / 0 / 4$ & $9 / 2 / 29$ & 0.581 \\
\hline Disease duration until VTE (months) & $86.8 \pm 109.6$ & $124.0 \pm 131.5$ & 0.153 \\
\hline History of smoking $(N, \%)$ & $3(10 \%)$ & $42(31.1 \%)$ & 0.045 \\
\hline History of VTE $(N, \%)$ & $3(9.3 \%)$ & $9(6.7 \%)$ & 0.702 \\
\hline \multicolumn{4}{|l|}{ Comorbidity } \\
\hline Malignancy & $1(3.1 \%)$ & $12(8.8 \%)$ & 0.466 \\
\hline $\mathrm{DM}$ & $1(3.1 \%)$ & $7(5.2 \%)$ & 1 \\
\hline HT & $3(9.3 \%)$ & $10(7.4)$ & 0.716 \\
\hline DLp & $1(3.1 \%)$ & $4(3.0 \%)$ & 1 \\
\hline Renal dysfunction & $0(0 \%)$ & $5(3.7 \%)$ & 0.584 \\
\hline Cardiovascular disease & $2(6.2 \%)$ & $6(4.4 \%)$ & 0.650 \\
\hline Site of VTE: L/PA/L + PA/PM/CVS/CR/ & $1 / 6 / 10 / 8 / 5 / 2$ & $44 / 11 / 16 / 23 / 3 / 38$ & $<0.0001$ \\
\hline Proportion of PA/PM/CVS & $28(87.5 \%)$ & $54(40.0 \%)$ & $<0.0001$ \\
\hline Situation at onset of VTE - hospitalization & $24(75.0 \%)$ & $96(71.1 \%)$ & 0.827 \\
\hline Hospitalization until onset of VTE (days) & $22.4 \pm 35.9$ & $18.2 \pm 14.5$ & 0.381 \\
\hline \multicolumn{4}{|l|}{ Clinical activity at onset of VTE } \\
\hline Partial Mayo (UC) & $5.4 \pm 3.2$ & $3.2 \pm 2.9$ & 0.0019 \\
\hline CDAI (CD) & $257.0 \pm 14.1$ & $227.6 \pm 97.7$ & 0.620 \\
\hline Patients with moderate to severe IBD activity $(N, \%)$ & $16(50.0 \%)$ & $39(29.5 \%)$ & 0.0079 \\
\hline Antithrombotic drugs before onset of VTE $(N, \%)$ & $1(3.1 \%)$ & $2(1.6 \%)$ & 1 \\
\hline \multicolumn{4}{|l|}{ Concomitant usage of treatment for IBD } \\
\hline $5-\operatorname{ASA}(N, \%)$ & $22(68.8 \%)$ & $87(64.4 \%)$ & 0.686 \\
\hline Corticosteroid $(N, \%)$ & $21(65.6 \%)$ & $67(49.6 \%)$ & 0.118 \\
\hline Immunomodulator $(N, \%)$ & $5(15.6 \%)$ & $28(20.7 \%)$ & 0.626 \\
\hline Calcineurin inhibitor $(N, \%)$ & $1(3.7 \%)$ & $9(6.6 \%)$ & 0.686 \\
\hline Anti-TNF antibody $(N, \%)$ & $1(3.1 \%)$ & $27(20.0 \%)$ & 0.018 \\
\hline Central venous catheter $(N, \%)$ & $15(46.8 \%)$ & $65(48.1 \%)$ & 1 \\
\hline Bowel resection $(N, \%)$ & $8(25.0 \%)$ & $44(32.6 \%)$ & 0.525 \\
\hline \multicolumn{4}{|l|}{ Laboratory data before onset of VTE } \\
\hline WBC $(/ \mu 1)$ & $9113 \pm 5189$ & $7866 \pm 4003$ & 0.153 \\
\hline $\mathrm{Hb}(\mathrm{g} / \mathrm{dl})$ & $10.9 \pm 1.93$ & $11.2 \pm 2.27$ & 0.451 \\
\hline Plt $\left(\times 10^{6} / \mu 1\right)$ & $30.3 \pm 20.0$ & $32.4 \pm 14.6$ & 0.508 \\
\hline Alb (g/dl) & $3.1 \pm 0.83$ & $3.2 \pm 0.83$ & 0.559 \\
\hline CRP (mg/dl) & $4.99 \pm 5.97$ & $2.60 \pm 3.84$ & 0.008 \\
\hline $\mathrm{CRP} \geq 1.5 \mathrm{mg} / \mathrm{dl}(N, \%)$ & $19(59.4 \%)$ & $49(36.3 \%)$ & 0.0266 \\
\hline D-dimer (ng/dl) & $9.06 \pm 15.6$ & $7.71 \pm 22.5$ & 0.844 \\
\hline FDP (ng/dl) & $30.0 \pm 48.1$ & $23.0 \pm 54.5$ & 0.727 \\
\hline
\end{tabular}


Table 4 continued

\begin{tabular}{|c|c|c|c|}
\hline Univariate analysis & Severity and death $(n=32)$ & Other $(n=135)$ & $p$ value \\
\hline AT-III (\%) & $88.1 \pm 21.5$ & $91.6 \pm 19.7$ & 0.639 \\
\hline $\begin{array}{l}\text { Multivariate analysis } \\
\text { Variables }\end{array}$ & \multicolumn{2}{|l|}{ OR $(95 \%$ CI $)$} & $p$ value \\
\hline Age at onset $\leq 45$ years & \multicolumn{2}{|c|}{$2.84(1.05-7.69)$} & 0.038 \\
\hline History of smoking & \multicolumn{2}{|c|}{$0.337(0.085-1.34)$} & 0.122 \\
\hline Site of VTE: PA/PM/CVS & \multicolumn{2}{|c|}{$9.79(3.03-31.6)$} & 0.00013 \\
\hline Moderate to severe IBD activity & \multicolumn{2}{|c|}{$2.79(1.04-7.47)$} & 0.0415 \\
\hline Usage of anti-TNF antibody & \multicolumn{2}{|c|}{$0.119(0.014-1.05)$} & 0.055 \\
\hline CRP before onset of VTE $\geq 1.5 \mathrm{mg} / \mathrm{dl}$ & \multicolumn{2}{|c|}{$2.13(0.78-5.26)$} & 0.148 \\
\hline
\end{tabular}

$B W$ body weight, $B M I$ body mass index, $I B D$ inflammatory bowel disease, $V T E$ venous thromboembolism, $U C$ ulcerative colitis, $C D$ Crohn's disease, $D M$ diabetes mellitus, $H T$ hypertension, $D L p$ dyslipidemia, $L$ deep venous thromboembolism in lower limbs, $P E$ pulmonary arterial thromboembolism, $C V S$ cerebral venous sinus thrombosis, $P V M$ portal and mesenteric venous thromboembolism, $C R$ catheter-related thrombosis, 5-ASA 5-amynosalicylate, TNF tumor necrosis factor, $I L$ interleukin, JAK Janus kinase, $W B C$ White blood cell count, $H b$ hemoglobin, $P l t$ platelet, $A l b$ albumin, $C R P$ C-reactive protein, FDP fibrin degradation product, $A T$-III antithrombin-III, $C I$ confidence interval, $O R$ odds ratio

Table 5 The outcomes after developing VTE

\begin{tabular}{lccc}
\hline \multicolumn{2}{l}{ Days until a serious condition developed from the onset of VTE (days) } & $3.3 \pm 6.3$ \\
\hline $\begin{array}{lll}\text { Days until death from onset of VTE (days) } \\
\text { Outcome of therapy for VTE }\end{array}$ & Total $(n=167)$ & Severity $(n=32)$ & Other $(n=135)$ \\
\hline improvement with elimination of VTE & $95(56.9 \%)$ & $17(53.1 \%)$ & $78(57.8 \%)$ \\
improvement with retention of VTE & $52(31.4 \%)$ & $7(21.9 \%)$ & $45(28.1 \%)$ \\
retention of sequelae & $6(3.6 \%)$ & $4(12.5 \%)$ & $2(1.5 \%)$ \\
Death & $4(2.4 \%)$ & $4(12.5 \%)$ & $0(0 \%)$ \\
Uncertain & $10(6.0 \%)$ & $0(0 \%)$ & $10(7.4 \%)$ \\
\hline
\end{tabular}

included than is typically encountered in most clinical settings in Japan. A further large-scale survey that includes municipal hospitals and clinics should be considered in the future.

In conclusion, this is the largest nationwide cohort study in Asia to describe the incidences of ATE and VTE and severe cases with death. The risk factors for severity and death were found to be the site of development of ATE and VTE, age, and disease activity. In addition, the therapeutic strategies and outcomes of ATE and VTE in IBD patients were described in this study. Although the incidence of ATE in Asian IBD patients was lower than that in reports from Western countries, the incidence of VTE was comparable between Asian and Western countries. Furthermore, the incidence of severe VTE was $20 \%$, with cases tending to show high risks of persistent VTE, developing sequelae, and dying. Therefore, an adequate risk classification system, screening, and prophylactic strategies for IBD-associated TE in Asian populations are urgently needed.
Supplementary InformationThe online version contains supplementary material available at https://doi.org/10.1007/s00535021-01829-5.

Acknowledgements We thank Shohei Oka, Yoichi Kakuta, Ryohei Hayashi, Daisuke Saito, Shinichiro Shinzaki, Eiko Saito, Maiko Kimura, Hideaki Hozumi, Ryota Hokari, Toshihiro Tanaka, Hiroki Tanaka, Toshiyuki Sakurai and Tomoya Iida for their great support of this study.

Authors' Contributions K.A. and M.F. contributed equally to this study. K.A. and M.F. provided major input into the conceptual development of the studies, analyzed the data, wrote the manuscript and supervised all of the investigations. K.A, M.F., K.W., S.H., H.S., S.T, H.I., T.M., T.K., M.N., H.I., S.K., T.T., K.K., R.H., T.F., T.K., M.E., S.Y., D.A., M.N., S.M., M.S., S.B., M.S., K.U., K.F., H.S., H.N., T.S., M.I., S.O., Y.K., H.R., D.S., S.S., E.S., M.K, H.H, R.H, T.T, H.T, T.S, and T.I. managed and treated the enrolled patients and collected the data. M.W., Y.S. and T.H. helped design the studies, interpret the data, and prepare/review the manuscript. All of the authors read and approved the final manuscript.

Funding The author(s) disclose receipt of the following financial support for the research, authorship, and/or publication of this article: This paper was supported by Intractable Disease Health and Labour 
Sciences Research Grants from the Ministry of Health, Labour and Welfare.

Data Availability Statement The data underlying this article will be shared on reasonable request to the corresponding author.

\section{Declarations}

Conflict of interest M.F. received lecture fees from Nippon Kayaku Co., Ltd., Janssen Pharmaceutical K.K., Takeda Pharmaceutical Co. Ltd., AbbVie GK., JIMRO Co. Ltd., AYUMI Pharmaceutical Corporation, and research grant from Nippon Kayaku Co. Ltd., Takeda Pharmaceutical Co. Ltd., Janssen Pharmaceutical K.K.,AbbVie GK., EA pharma Co. Ltd., AYUMI Parmaceutical Industries Co. Ltd. K.W. received (1) lecture fees from Abbvie GK., EA Pharma Co.,Ltd., Kissei Pharmaceutical Co., Ltd., Pfizer Lnc.,Kyorin Pharmaceutical Co.,Ltd., Mitsubishi Tanabe Pharma Corporation, Janssen Pharmaceutical K.K, Takeda Pharmaceutical Co.,Ltd., Zeria Pharmaceutical Co.,Ltd., Mochida Pharmaceutical Co.,Ltd., (2) research and scholarship grants from EA Pharma Co.,Ltd., Takeda Pharmaceutical Co.,Ltd., EP-CRSU Co. Ltd., and (3) endowed chair from AbbVie GK., EA pharma Co. Ltd., Mitsubishi Tanabe Pharma Co., Zeria Pharmaceutical Co.,Ltd., Asahi Kasei Medical Co.,Ltd., and Mochida Pharmaceutical Co.,Ltd. S.H. received lecture fees from Janssenn Pharaceutical K.K., Mitsubishi Tanabe Pharma Co. T.M. received endowed chair from Kinshukai Medical Corporation. T.K. received lecture fees AbbVie GK., Alfresa Pharma Co., Janssen Pharmaceutical K.K., Takeda Pharmaceutical Co. Ltd., Mitsubishi Tanabe Pharma Co., Pfizer Lnc., Mochida Pharmaceutical Co.,Ltd., and research grants from Nippon Kayaku Co., Ltd. and EA pharma Co. Ltd., and scholarship grants from Otsuka Holdings, JIMRO Co. Ltd., EA pharma Co. Ltd., AbbVie GK and Zeria Pharmaceutical Co.,Ltd. and Pfizer Lnc. M.N. received lecture fees from Takeda Pharmaceutical Co. Ltd., and research grant from Hitachi Ltd. S.K. Received lecture fees from AbbVie GK., Janssen Pharmaceutical K.K. and Mitsubishi Tanabe Pharma Co., and scholarship grants from AbbVie GK. and EA pharma Co. Ltd. T.T received research grants from Mitsubishi Tanabe Pharma Corporation, EA Pharma Co., AbbVie GK., Zeria Pharmaceutical Co., Ltd. M.N. received lecture fees from Takeda Pharmaceutical Co. Ltd., AbbVie GK., Mitsubishi Tanabe Pharma Co. and Pfizer Inc., and research grant from Mochida Pharmaceutical Co. Ltd., and scholarship grants from EA Pharma Co., AbbVie GK. and Mitsubishi Tanabe Pharma Co. H.N. received lecture fees from AbbVie GK., Kissei Pharmaceutical Co. Ltd., Kyorin Pharmaceutical Co. Ltd., Mitsubishi Tanabe Pharma Co., Janssen Pharmaceutical K.K., Takeda Pharmaceutical Co. Ltd., Pfizer Inc., Celgene Co. and EA pharma Co. Ltd., and research grants from HOYA Group Pentax Medical, Mitsubishi Tanabe Pharma Co., Pfizer Lnc., AbbVie GK and Mochida Pharmaceutical Co., and scholarship grants from AbbVie GK, Mitsubishi Tanabe Pharma Co., Takeda Pharmaceutical Co. Ltd., Nippon Kayaku Co. Ktd., Otsuka Pharmaceutical Co. Ltd. and EA Pharma Co. Ltd. T.H. received lecture fees from Mitsubishi Tanabe Pharma Corporation, AbbVie GK, EA pharma Co. Ltd., Kyorin Pharmaceutical Co. Ltd., JIMRO Co., Janssen Pharmaceutical K.K., Mochida Pharmaceutical Co., Ltd., Takeda Pharmaceutical Co. Ltd., consulting fees from EA pharma Co. Ltd., AbbVie GK, Celgene K.K., Janssen Pharmaceutical K.K., Pfizer Inc., Nichi-Iko Pharmaceutical Co., Ltd. and research grant from Alfresa Pharma Co. Ltd., EA pharma Co. Ltd., scholarship grants from Mitsubishi Tanabe Pharma Corporation, EA pharma Co. Ltd.AbbVie GK, JIMRO Co. Ltd., Zeria Pharmaceutical Co. Ltd., Daiichi-Sankyo, Kyorin Pharmaceutical Co. Ltd., Nippon Kayaku Co. Ltd., Takeda Pharmaceutical Co. Ltd., Pfizer Inc., Mochida Pharmaceutical Co., Ltd.
Open Access This article is licensed under a Creative Commons Attribution 4.0 International License, which permits use, sharing, adaptation, distribution and reproduction in any medium or format, as long as you give appropriate credit to the original author(s) and the source, provide a link to the Creative Commons licence, and indicate if changes were made. The images or other third party material in this article are included in the article's Creative Commons licence, unless indicated otherwise in a credit line to the material. If material is not included in the article's Creative Commons licence and your intended use is not permitted by statutory regulation or exceeds the permitted use, you will need to obtain permission directly from the copyright holder. To view a copy of this licence, visit http://creativecommons. org/licenses/by/4.0/.

\section{References}

1. Zezos P, Kouklakis G, Saibil F. Inflammatory bowel disease and thromboembolism. World J Gastroenterol. 2014;20:13863-78.

2. Kohoutova D, Moravkova P, Kruzliak P, et al. Thromboembolic complications in inflammatory bowel disease. $\mathbf{J}$ Thromb Thrombolysis. 2015;39:489-98.

3. Miehsler W, Reinisch W, Valic E, et al. Is inflammatory bowel disease an independent and disease specific risk factor for thromboembolism? Gut. 2004;53:542-8.

4. Grainge MJ, West J, Card TR. Venous thromboembolism during active disease and remission in inflammatory bowel disease: a cohort study. The Lancet. 2010;375:657-63.

5. Weissman S, Sinh P, Mehta TI, et al. Atherosclerotic cardiovascular disease in inflammatory bowel disease: The role of chronic inflammation. World J Gastrointest Pathophysiol. 2020;11:104-13.

6. Giannotta M, Tapete G, Emmi G, et al. Thrombosis in inflammatory bowel diseases: what's the link? Thromb J. 2015;13:14.

7. Nguyen GC, Bernstein CN, Bitton A, et al. Consensus statements on the risk, prevention, and treatment of venous thromboembolism in inflammatory bowel disease: Canadian association of gastroenterology. Gastroenterology. 2014;146:835-848.e6.

8. Fumery M, Xiaocang C, Dauchet L, et al. Thromboembolic events and cardiovascular mortality in inflammatory bowel diseases: A meta-analysis of observational studies. J Crohn and Colitis. 2014;8:469-79.

9. Kirchgesner J, Beaugerie L, Carrat F, et al. Increased risk of acute arterial events in young patients and severely active IBD: A nationwide French cohort study. Gut. 2018;67:1261-8.

10. Ghoneim S, Shah A, Dhorepatil A, et al. The risk of cerebrovascular accidents in inflammatory bowel disease in the United States: A population-based national study. Clin Exp Gastroenterol. 2020;13:123-9.

11. Singh S, Singh H, Loftus EV, et al. Risk of cerebrovascular accidents and ischemic heart disease in patients with inflammatory bowel disease: A systematic review and meta-analysis. Clin Gastroenterol Hepatol. 2014;12:382-93.

12. Andrade AR, Barros LL, Azevedo MFC, et al. Risk of thrombosis and mortality in inflammatory bowel disease. Clin Transl Gastroenterol. 2018;9:142.

13. Solem CA, Loftus EV, Tremaine WJ, et al. Venous thromboembolism in inflammatory bowel disease. Am J Gastroenterol. 2004;99:97-101.

14. Talbot RW, Heppell J, Dozois RR, et al. Vascular Complications of Inflammatory Bowel Disease. Mayo Clin Proc. 1986;61:140-5.

15. Nguyen GC, Sam J. Rising prevalence of venous thromboembolism and its impact on mortality among hospitalized inflammatory bowel disease patients. Am $\mathrm{J}$ of Gastroenterol. 2008;103:2272-80. 
16. Dorn SD, Sandler RS. Inflammatory bowel disease is not a risk factor for cardiovascular disease mortality: Results from a systematic review and meta-analysis. Am J Gastroenterol. 2007;102:662-7.

17. Kassam Z, Belga S, Roifman I, et al. Inflammatory bowel disease cause-specific mortality: a primer for clinicians. Inflamm Bowel Dis. 2014;20:2483-92.

18. Romberg-Camps M, Kuiper E, Schouten L, et al. Mortality in inflammatory bowel disease in the Netherlands 1991-2002: Results of a population-based study: The IBD South-Limburg cohort. Inflamm Bowel Dis. 2010;16:1397-410.

19. Ha C, Magowan S, Accortt NA, et al. Risk of arterial thrombotic events in inflammatory bowel disease. Am J Gastroenterol. 2009;104:1445-51.

20. Weng MT, Park SH, Matsuoka K, et al. Incidence and risk factor analysis of thromboembolic events in east asian patients with inflammatory bowel disease, a multinational collaborative study. Inflamm Bowel Dis. 2018;24:1791-800.

21. Ueshima H, Sekikawa A, Miura K, et al. Cardiovascular Disease and Risk Factors in Asia A Selected Review. Circulation. 2008;118:2702-9.

22. Wang KL, Yap ES, Goto S, et al. The diagnosis and treatment of venous thromboembolism in asian patients. Thromb J. 2018;16(1).

23. Harbord M, Annese V, Vavricka SR, et al. The first european evidence-based consensus on extra-intestinal manifestations in inflammatory bowel disease. J Crohn Colitis. 2016;10:239-54.

24. Kornbluth A, Sachar DB. Ulcerative colitis practice guidelines in adults: American college of gastroenterology, practice parameters committee. Am J Gastroenterol. 2010;105:500.

25. Kubo M, Kiyohara Y, Kato I, et al. Trends in the incidence, mortality, and survival rate of cardiovascular disease in a japanese community the hisayama study. Stroke. 2003;34:2349-54.

26. Global health estimates: Leading causes of death. https://www. who.int/data/gho/data/themes/mortality-and-global-health-esti mates/ghe-leading-causes-of-death

27. Sakuma M, Nakamura M, Yamada N, et al. Venous thromboembolism: -deep vein thrombosis with pulmonary embolism, deep vein thrombosis alone, and pulmonary embolism alone-. Circ J. 2009;73:305-9.

28. Lee LH, Gallus A, Jindal R, et al. Incidence of venous thromboembolism in asian populations: a systematic review. Thrombo Haemost. 2017;117:2243-60.

29. Kappelman MD, Horvath-Puho E, Sandler RS, et al. Thromboembolic risk among Danish children and adults with inflammatory bowel diseases: a population-based nationwide study. Gut. 2011;60:937-43.
30. Bernstein CN, Blanchard JF, Houston DS, et al. The incidence of deep venous thrombosis and pulmonary embolism among patients with inflammatory bowel disease: a population-based cohort study. Thromb Haemost. 2001;85:430-4.

31. Huerta C, Johansson S, Wallander MA, et al. Risk factors and short-term mortality of venous thromboembolism diagnosed in the primary care setting in the United Kingdom. Arch Intern Med. 2007;167:935-43.

32. Ando K, Fujiya M, Nomura $\mathrm{Y}$, et al. The incidence and risk factors of venous thromboembolism in Japanese inpatients with inflammatory bowel disease: A retrospective cohort study. Intest Res. 2018;16:416-25.

33. Ando K, Fujiya M, Nomura $\mathrm{Y}$, et al. The incidence and risk factors of venous thromboembolism in patients with inflammatory bowel disease: a prospective multicenter cohort study. Digestion. 2019;100:229-37.

34. Ohta Y, Arai M, Nakagawa T, et al. Comparison of a novel predictor of venous thromboembolic complications in inflammatory bowel disease with current predictors. J Gastroenterol Hepatol. 2019;34:870-9.

35. Sonoda K, Ikeda S, Mizuta Y, et al. Evaluation of venous thromboembolism and coagulation-fibrinolysis markers in Japanese patients with inflammatory bowel disease. J Gastroenterol. 2004;39:948-54.

36. Itabashi $\mathrm{M}$, Ikeuchi $\mathrm{H}$, Kimura $\mathrm{H}$, et al. Perioperative venous thromboembolism in ulcerative colitis: a multicenter prospective study in Japan. Crohns Colitis 360. 2021;360(3):1-7.

37. Danese S, Papa A, Saibeni S, et al. Inflammation and coagulation in inflammatory bowel disease: The clot thickens. Am J Gastroenterol. 2007;102:174-86.

38. Osnes LTN, Foss KB, Joø GB, et al. Acetylsalicylic acid and sodium salicylate inhibit LPS-induced NF- $\kappa B / c-$ rel nuclear translocation, and synthesis of tissue factor (TF) and tumor necrosis factor alfa (TNF- $\alpha$ ) in human monocytes. Thrombo Haemost. 1996;76:970-6.

39. Kirchgesner J, Nyboe Andersen N, Carrat F, et al. Risk of acute arterial events associated with treatment of inflammatory bowel diseases: Nationwide French cohort study. Gut. 2020;69:852-8.

40. le Gall G, Kirchgesner J, Bejaoui M, et al. Clinical activity is an independent risk factor of ischemic heart and cerebrovascular arterial disease in patients with inflammatory bowel disease. PLoS ONE. 2018;13:e201991.

Publisher's Note Springer Nature remains neutral with regard to jurisdictional claims in published maps and institutional affiliations. 